\title{
Resveratrol-Potential Antibacterial Agent against Foodborne Pathogens
}

\section{OPEN ACCESS}

Edited by:

Lyndy Joy McGaw.

University of Pretoria, South Africa

Reviewed by:

Susan Semple,

University of South Australia, Australia

Ligia Salgueiro,

University of Coimbra, Portugal

*Correspondence:

Tahir Mehmood Khan tahir.khan@uvas.edu.pk

Learn-Han Lee

lee.learn.han@monash.edu; leelearnhan@yahoo.com Bey-Hing Goh

goh.bey.hing@monash.edu

Specialty section:

This article was submitted to

Ethnopharmacology,

a section of the journal

Frontiers in Pharmacology

Received: 23 October 2017 Accepted: 30 January 2018 Published: 19 February 2018

Citation:

Ma DSL, Tan LT-H, Chan K-G, Yap WH, Pusparajah P, Chuah L-H, Ming LC, Khan TM, Lee L-H and Goh B-H

(2018) Resveratrol-Potential Antibacterial Agent against Foodborne Pathogens. Front. Pharmacol. 9:102 doi: 10.3389/fphar.2018.00102

\author{
Dexter S. L. Ma ${ }^{1,2}$, Loh Teng-Hern Tan ${ }^{1,2,3}$, Kok-Gan Chan ${ }^{4,5}$, Wei Hsum Yap ${ }^{6}$, \\ Priyia Pusparajah ${ }^{3}$, Lay-Hong Chuah ${ }^{1,2,7}$, Long Chiau Ming ${ }^{8,9}$, Tahir Mehmood Khan 1,2,10,11*, \\ Learn-Han Lee ${ }^{1,2,10,12 *}$ and Bey-Hing Goh ${ }^{1,2,10,12 *}$
}

${ }^{1}$ Biofunctional Molecule Exploratory Research Group, School of Pharmacy, Monash University Malaysia, Bandar Sunway, Malaysia, ${ }^{2}$ Novel Bacteria and Drug Discovery Research Group, School of Pharmacy, Monash University Malaysia, Bandar Sunway, Malaysia, ${ }^{3}$ Biomedical Research Laboratory, Jeffrey Cheah School of Medicine and Health Sciences, Monash University Malaysia, Subang Jaya, Malaysia, ${ }^{4}$ International Genome Centre, Jiangsu University, Zhenjiang, China, ${ }^{5}$ Division of Genetics and Molecular Biology, Faculty of Science, Institute of Biological Sciences, University of Malaya, Kuala Lumpur, Malaysia, ${ }^{6}$ School of Biosciences, Taylor's University, Subang Jaya, Malaysia, ${ }^{7}$ Advanced Engineering Platform, Monash University Malaysia, Subang Jaya, Malaysia, ${ }^{8}$ Division of Pharmacy, School of Medicine, University of Tasmania, Hobart, Australia, ${ }^{9}$ School of Pharmacy, KPJ Healthcare University College, Nilai, Malaysia, ${ }^{10}$ Asian Centre for Evidence Synthesis in Population, Implementation and Clinical Outcomes, Health and Well-Being Cluster, Global Asia in the 21st Century Platform, Monash University Malaysia, Subang Jaya, Malaysia, ${ }^{11}$ The Institute of Pharmaceutical Sciences, University of Veterinary and Animal Sciences, Lahore, Pakistan, ${ }^{12}$ Center of Health Outcomes Research and Therapeutic Safety, School of Pharmaceutical Sciences, University of Phayao, Phayao, Thailand

Bacterial foodborne pathogens are a significant health burden and the recent emergence of pathogenic resistant strains due to the excessive use of antibiotics makes it more difficult to effectively treat infections as a result of contaminated food. Awareness of this impending health crisis has spurred the search for alternative antimicrobials with natural plant antimicrobials being among the more promising candidates as these substances have good acceptability and likely low toxicity levels as they have long been used in traditional medicines. Resveratrol (3,5,4'-trihydroxystilbene) is a naturally occurring stilbenoid which has been gaining considerable attention in medical field due to its diverse biological activities - it has been reported to exhibit antioxidant, cardioprotective, anti-diabetic, anticancer, and antiaging properties. Given that resveratrol is phytoalexin, with increased synthesis in response to infection by phytopathogens, there has been interest in exploring its antimicrobial activity. This review aims to provide an overview of the published data on the antibacterial activity of resveratrol against foodborne pathogens, its mechanisms of action as well as its possible applications in food packing and processing; in addition we also summarize the current data on its potential synergism with known antibacterials and future research and applications.

Keywords: resveratrol, antibacterial, foodborne, pathogens, antibiofilm

\section{INTRODUCTION}

Foodborne illnesses resulting from of ingestion of food or drinks contaminated with pathogenic microorganisms represent a global health concern (Lv et al., 2011). Transmission of foodborne pathogens from contaminated food is a common health concern, the causative agents from bacteria, viruses, parasites, chemicals and prions. Kirk et al. (2015) estimated there were 2 billion cases of foodborne diseases in 2010 alone and over 1 million deaths with 78.7 million Disability Adjusted 
Life Years (DALYs) reported. About 582 million foodborne diseases cases were due to transmission from contaminated food, and the leading causative agent of foodborne illness was Norovirus which caused 125 million cases whereas Campylobacter spp. caused 96 million illness. The highest burden was diarrhea due to infection by non-typhoidal Salmonella enterica (NTS) which resulted in 4.07 million DALYs (Kirk et al., 2015).

The continuous use of antimicrobial agents like antibiotics is one of the main reasons antibiotic resistant bacteria strains have developed. Overuse of antibiotics in agriculture where they are used not only for treatment of infections but also for promoting animal growth is a significant public health risk as it promotes the of emergence and spread of antibiotic resistant bacteria from food producing animals (Tan et al., 2014). For example, the use of beta-lactams and ciprofloxacin resulted in the emergence of resistant strains of Campylobacter spp. and the use of streptomycin, sulfisoxazole and tetracycline resulted in development of resistance in Escherichia coli (Das et al., 2017). Since the introduction of tetracycline in 1957, the proportion of tetracycline resistant E. coli isolated from poultry increased from 3.5 to $63.2 \%$ in United Kingdom from 1957 to 1961 (Tellez et al., 2013). In short, the rise in antibiotic resistance has led to a decrease in the effectiveness of many antibiotics-a scenario which has tremendous implications for the pharmaceutical, medical, and food industries.

Given the significant morbidity and mortality associated with foodborne pathogens, the pharmaceutical industry is investing heavily in research looking for new inhibitory compounds from natural sources that can be developed into new anti-microbial drugs. Plant based medications have deep roots in medicinal lore, with mankind having relied on natural products derived from plants, animals, and microbes as remedies to treat illnesses for a very long time (Tan et al., 2015, 2017; Tang et al., 2016; Chan et al., 2017; Ser et al., 2017). In fact, in current clinical medicine, many modern drugs actually originated from plants which are rich in chemically diverse compounds and scaffolds (Fabricant and Farnsworth, 2001; Ser et al., 2016; Yong et al., 2017). In recent years, there has been increased interest in research seeking plant derived antimicrobials as an alternative to antibiotics. In addition to their efficacy in terms of antimicrobial properties, a significant advantage of these substances is that bacteria are less likely to develop resistance against them as they are acting on more than one target in cells (Burt, 2004). Also, certain plant derived antimicrobials are labeled as Generally Recognized as Safe (GRAS), giving them a "greener" image that is more acceptable to consumers (Xi et al., 2011). All these factors in combination suggest that natural antimicrobials have significant healthcare and market value given they match the increase in demand of greener additives by the consumers, supporting the value of research of plant based antimicrobials as potential biopharmaceutical products (Lai et al., 2017).

Among the various groups of plant based natural products, resveratrol (3,5, $4^{\prime}$-trihydroxystilbene) seems highly promising for its therapeutic applications. It is a compound present in grape skins and wine, and is known to exhibit multispectrum therapeutic applications having been reported to exhibit anticancer, antiangiogenic, immunomodulatory, cardioprotective, and antioxidant activity. Although there is still very limited work on the antibacterial activity of resveratrol, it has been shown that resveratrol exhibits antibacterial activity against several Gram-positive and Gram-negative foodborne bacteria (Chan, 2002; Tegos et al., 2002; Paulo et al., 2010; Paolillo et al., 2011; Alvarez et al., 2012, 2013; Kumar et al., 2012b; Plumed-Ferrer et al., 2013; Augustine et al., 2014; Ferreira et al., 2014; Moran et al., 2014; Promgool et al., 2014; Subramanian et al., 2014; Duarte et al., 2015; Kim et al., 2015; Makwana et al., 2015; Ferreira and Domingues, 2016; Liu et al., 2016; Seukep et al., 2016; Silva et al., 2016; Surendran Nair et al., 2016; Klancnik et al., 2017; Lai et al., 2017; Lee and Lee, 2017; Oliveira et al., 2017). This review aims to provide an overview of the published data on the antibacterial activity of resveratrol against foodborne pathogens, its mechanisms of action as well as its possible applications in food packing and processing; in addition we also summarize the current data on its potential synergism with known antibacterials and future research and applications. Figure 1 depicts the overview of this review.

\section{OVERVIEW AND RATIONALE OF RESVERATROL AS ANTIMICROBIAL AGENT}

Resveratrol is a naturally occurring phytoalexin belonging to the stilbene family of phenolic compounds; it exists in either trans or cis geometrical isomers with the trans isomer (Figure 2) being the predominant isomer in plants (Filip et al., 2003; Catalgol et al., 2012) and hence forming the focus of this review. First isolated by Takaoka in 1940 from the roots of white hellebore (Veratrum grandiflorum O. Loes), the compound was later named resveratrol since it is a resorcinol derivative from Veratrum species (Catalgol et al., 2012). As a polyphenolic compound, resveratrol has been reported in over 100 medical and edible plants such as Polygonum cuspidatum, Arachis hypogaea, Yucca Shidigera, Cassia quinquangulata, Rheum rhamponticum, and many more (Rocha-Gonzalez et al., 2008; Chachay et al., 2011). In 1992, resveratrol, as the active component in red wine, was believed to be responsible for the phenomenon known as the "French paradox" whereby epidemiological data shows that the French population have a lower mortality rate from cardiovascular diseases (CHD) despite a relatively high dietary fat consumption (Catalgol et al., 2012; Tome-Carneiro et al., 2013). Renaud and de Lorgeril (1992) proposed that the lower mortality rate of the French population-in spite of their relatively fatty diet-from $\mathrm{CHD}$ was due to the moderate consumption of resveratrol-containing wine, and suggested that the consumption of wine inhibited platelet aggregation thus leading to reduced risk of CHD (Renaud and de Lorgeril, 1992). In the following years, resveratrol was very much in the spotlight with the first evidence published of it potential to prevent carcinogenesis in mice (Jang et al., 1997). Since then, resveratrol has received considerable attention for its health promoting properties such as its antioxidant, cardioprotective, anti-diabetic, anticancer, and antiaging properties (Jung et al., 2009; Fernández-Mar et al., 2012). 


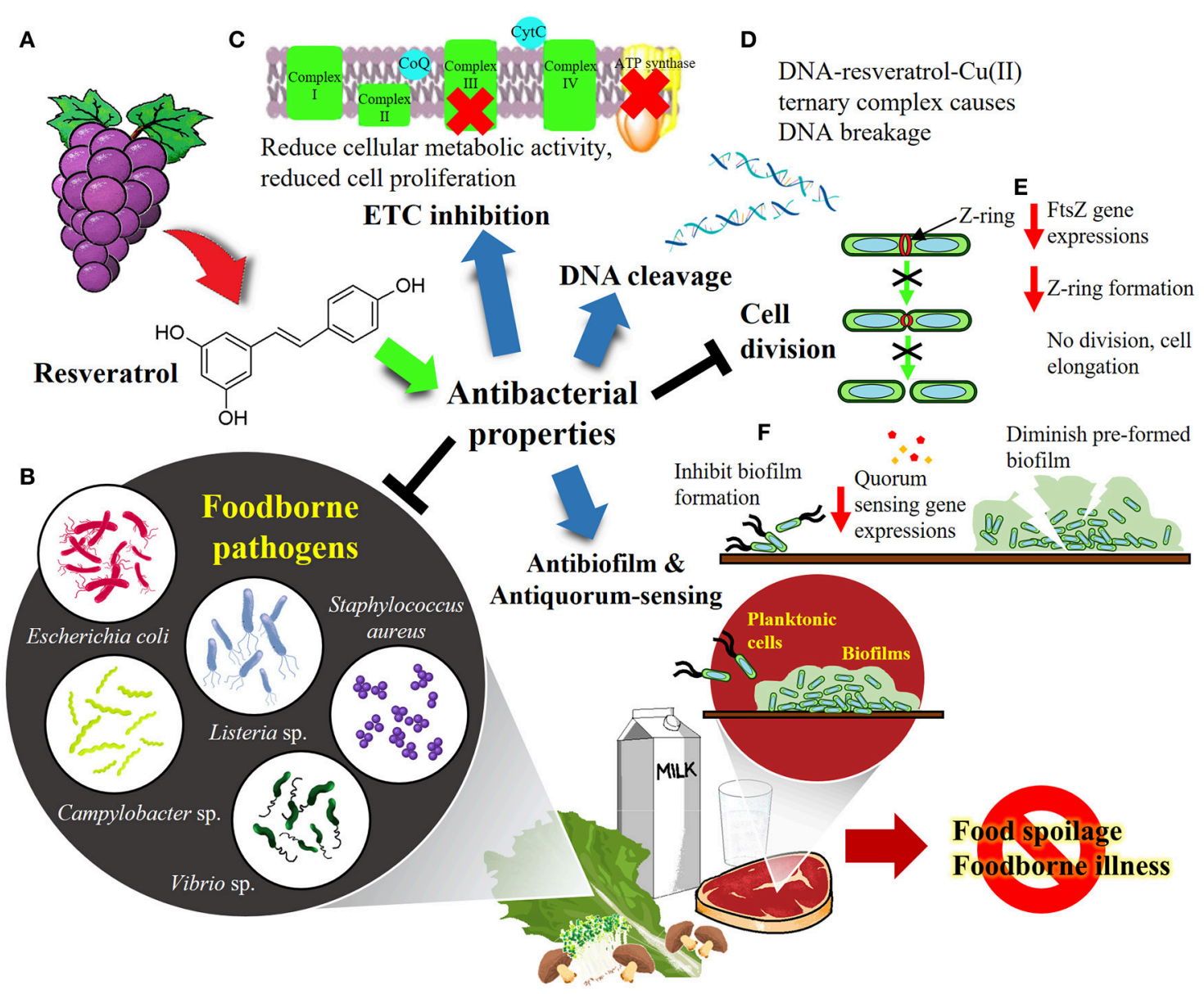

FIGURE 1 | The overview of resveratrol as antibacterial agent against foodborne pathogens. (A) Resveratrol is found abundantly in the skin and leaves of grapevine (Vitis vinifera). (B) Resveratrol has been shown to exhibit promising antibacterial properties against variety of foodborne pathogens such as E. coli, Listeria sp., Staphylococcus aureus, Campylobacter sp. and Vibrio sp. (C) Resveratrol was shown to inhibit electron transport chain (ETC) and FOF1-ATPase, leading to reduced cellular energy production and thus proliferation of the microorganisms. (D) Resveratrol binds to Cu(II) to form a Cu(II)-peroxide complex, which binds DNA to form DNA-resveratrol-Cu(II) ternary complex, leading to DNA scission and breakage. (E) Resveratrol inhibits cell division by downregulating FtsZ gene which responsible for the formation of Z-ring required for prokaryotic cell division. (F) Resveratrol was demonstrated to be an effective antibiofilm and antiquorum sensing agent that prevents biofilm formation as well as disrupts the pre-formed biofilm. Overall, resveratrol could be developed into biopharmaceutical product as well as food preservatives in food industry to cope with food spoilage and foodborne outbreaks.<smiles>Oc1ccc(/C=C/c2cc(O)cc(O)c2)cc1</smiles>

trans-resveratrol
B<smiles>Oc1ccc(/C=C\c2cc(O)cc(O)c2)cc1</smiles>

cis-resveratrol

FIGURE 2 | Chemical structures of (A) trans-resveratrol and (B) cis-resveratrol.

Phytoalexins are naturally-occurring, low molecular weight secondary metabolites produced by plants in respond to adverse conditions such as biotic stress from bacteria, fungi, viruses, and abiotic stress from chemical treatment (Filip et al., 2003; Pangeni et al., 2014). For instance, resveratrol is known as a major stilbene phytoalexin which is produced as an active defense 
mechanism to protect grape plants from phytopathogens. During Botrytis cinerea fungal infection, the amount of resveratrol was found to increase in the skin of infected grapes (Jeandet et al., 1995). Moreover, the synthesis of resveratrol was also increased in grapevine berries during fungal infection by Aspergillus carbonarius (Bavaresco et al., 2008). Similarly, the bacterium belonging to genus Bacillus was also shown to stimulate the production of resveratrol in the leaves of grapevine plants (Paul et al., 1998). Besides that, the grapevine Vitis vinifera which is rich in resveratrol has also been used as popular folk remedy to treat diarrhea, hemorrhage, varicose veins, inflammatory disorder and also heal wounds and drain furuncles (Bombardelli and Morazzoni, 1995; Pari and Suresh, 2008; Orhan et al., 2009). Resveratrol, as a logical target, has been investigated in the same manner as other plant derived phenolic compounds (Chan et al., 2016), in many previous studies as alternatives to antibiotics (Burt, 2004). The work so far appears encouraging with resveratrol having been reported to exhibit antimicrobial properties against bacteria (Hwang and Lim, 2015), yeasts (Jung et al., 2007) and fungi (Jung et al., 2005).

\section{IN VITRO ANTIBACTERIAL TESTS OF RESVERATROL}

Several analytical methods have been employed to evaluate the antibacterial activity of resveratrol against foodborne bacterial pathogens; with the two most important methods used being the disk diffusion assay and broth microdilution assay which enable an estimate of the efficacy of resveratrol against a tested pathogen. Furthermore, several other advanced techniques have also been adopted to evaluate the antibacterial activity of resveratrol such as time kill analysis, scanning electron microscopy, crystal violet biofilm assay, and quorum sensing inhibition assay (Burt, 2004; O’toole, 2011). Based on the available literature, resveratrol was demonstrated to exhibit differential antibacterial activities against different strains of foodborne pathogens including Staphylococcus aureus, Bacillus cereus, Bacillus subtilis, and Listeria monocytogenes, E. coli O157:H7, Salmonella Typhimurium, Vibrio cholera, Campylobacter jejuni, Campylobacter coli, Arcobacter butzleri, and Arcobacter cryaerophilus (Tables 1, 2).

According to the data from previous studies, the minimum inhibitory concentration (MIC) of resveratrol against Gramnegative ranged from $0.625-521 \mu \mathrm{g} / \mathrm{mL}$ while the MIC of resveratrol against Gram-positive bacteria ranged from 16.5$260 \mu \mathrm{g} / \mathrm{mL}$. In general, previous studies indicated that resveratrol exhibits better activity against Gram-positive bacteria than Gram-negative bacteria. Using a simple analysis based on the data collected, it was found that the mean MIC of resveratrol against Gram-positive bacteria was $141.83 \pm 18.30 \mu \mathrm{g} / \mathrm{ml}$ while against Gram-negative bacteria was $224.82 \pm 39.41 \mu \mathrm{g} / \mathrm{ml}$. For instance, Paulo et al. (2010) reported that the MIC of resveratrol toward all tested Gram-negative bacteria were more than $400 \mu \mathrm{g} / \mathrm{mL}$ while MIC toward Gram-positive bacteria ranged from 50 to $100 \mu \mathrm{g} / \mathrm{mL}$. They proposed that the difference in susceptibilities toward resveratrol might be attributed to the presence of a hydrophilic outer membrane in Gram-negative bacteria which is absent in Gram-positive bacteria. The hydrophilic outer membrane may act as a protective layer resisting the diffusion of hydrophobic molecules like resveratrol from penetrating the bacterial cell, thereby reducing the efficacy of the antibacterial action of resveratrol (Paulo et al., 2010). Besides that, the presence of degradative and detoxifying enzymes in the periplasmic space of Gram-negative bacteria could be another contributing factor to reduced activity of resveratrol due to breakdown of the resveratrol molecule by these enzymes (Beveridge, 1999). Another possibility of the observed lower sensitivity demonstrated by Gram-negative bacteria toward resveratrol could be the presence of multidrug resistance pumps (MDRPs) on their cell surface which may be able to extrude resveratrol from the cell. This appears to be unique to Gram negative rather than Gram positive organisms as demonstrated by Tegos et al. (2002) who reported that the MIC of resveratrol for S. aureus and E. coli was $125 \mu \mathrm{g} / \mathrm{mL}$ and $500 \mu \mathrm{g} / \mathrm{ml}$ respectively. However, the MIC of resveratrol for E. coli decreased by 16 -fold in the presence of a MDRPs inhibitor while for $S$. aureus, the MIC only decreased by 2 -fold which suggested the effect of MDRPs in reducing the activity of resveratrol in Gram-negative bacteria (Tegos et al., 2002). Nevertheless, it is still difficult to draw any definite conclusions about the difference in susceptibility between Grampositive and Gram-negative bacteria toward resveratrol. A study by Taguri et al. (2006) demonstrated that the susceptibility to phenolic compounds was dependent on the bacterial species with Gram-staining not necessarily correlating with the antimicrobial efficacy.

Combinatorial therapy may be one strategy to address the differential susceptibility toward resveratrol and development of resistance, as it could potentially enhance the efficacy of antimicrobials as well as to prevent emergence of resistant strains by exploiting the synergism between multiple antimicrobials. Kumar et al. (2012a) reported the existence of synergistic activity of resveratrol and antibiotics, showing improved antibacterial activity compared to treatment of resveratrol or the antibiotic alone. The study revealed the synergism between resveratrol and ciprofloxacin while additive effect between resveratrol and cefotaxime against both Gram-negative or Gram-positive bacteria (Kumar et al., 2012a). It may be pertinent for future research to investigate the interactions between resveratrol with other commonly used antibiotics in clinical medicine in order to fully exploit resveratrol as a novel antibacterial agent.

\section{POSSIBLE MODE OF ACTIONS OF RESVERATROL AGAINST FOODBORNE BACTERIA}

Understanding the mechanisms underlying the actions of a potential antibacterial molecule is key to assisting in identifying the likely targets and predicting the possible outcomes of resistance mechanisms that target bacteria may develop toward the molecule. The exact mechanism of the antibacterial activities of resveratrol against bacterial pathogens are still elusive. Nevertheless, several studies have reported possible antibacterial 
TABLE 1 | MIC of resveratrol against Gram-negative bacteria.

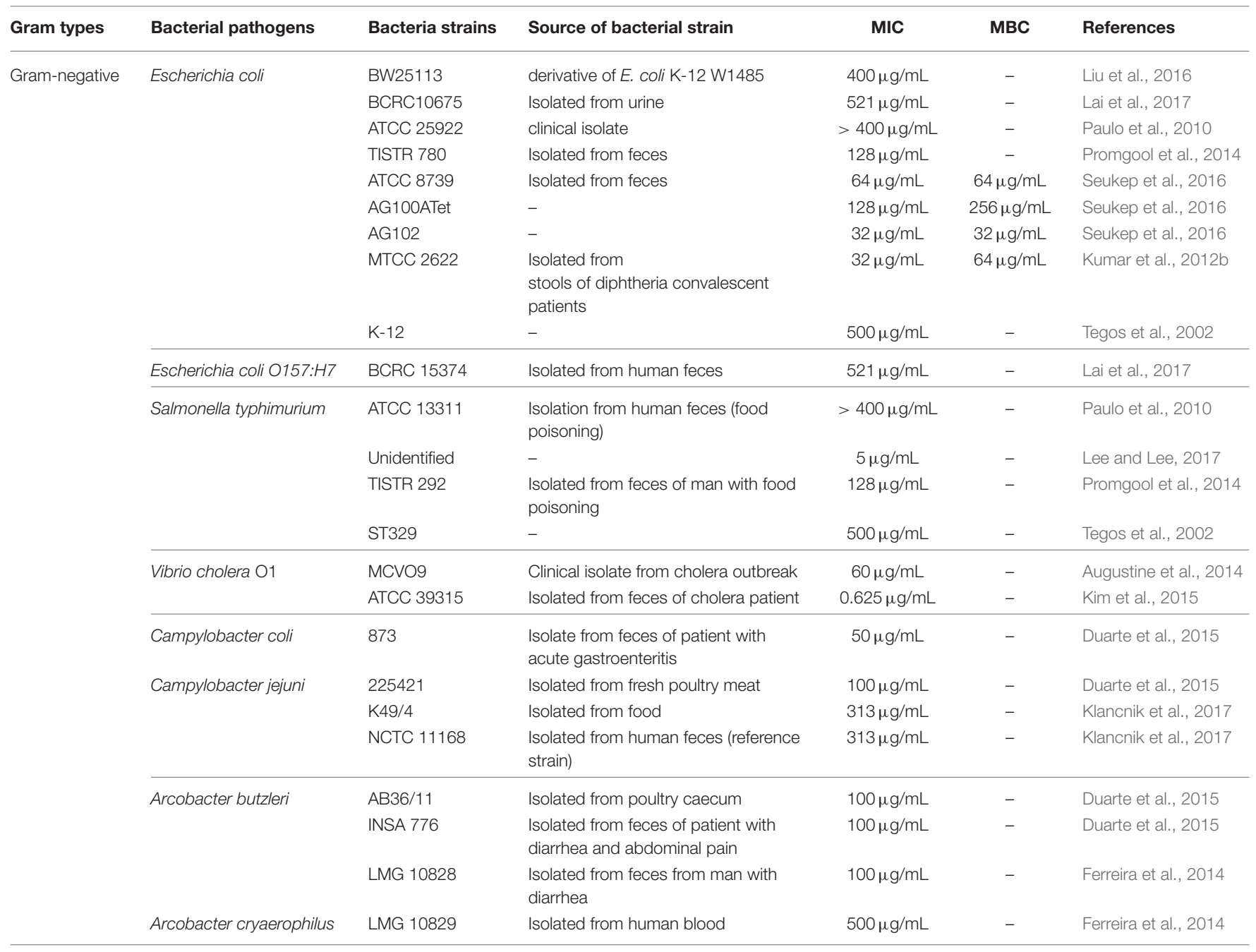

TABLE 2 | MIC of resveratrol against Gram-positive bacteria.

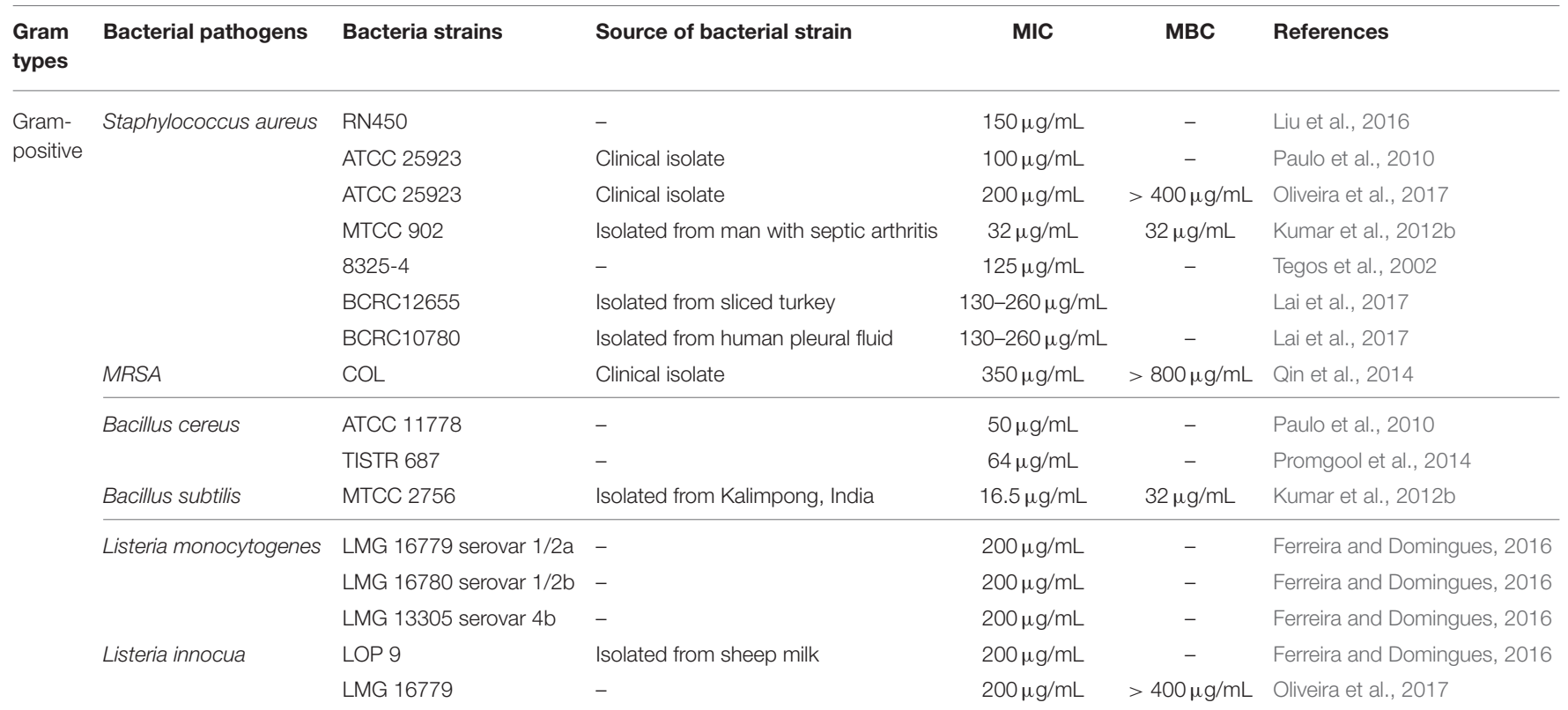


mechanism of action of resveratrol which include DNA damage (Subramanian et al., 2016), cell division impairment (Haranahalli et al., 2016), oxidative membrane damage (Subramanian et al., 2014), and also metabolic enzymes inhibition (Dadi et al., 2009).

Many antibiotics induce oxidative stress in target bacteria by producing ROS that cause oxidative damage to DNA, subsequently leading to cell death (Goswami et al., 2006). Even though the antioxidant properties of resveratrol are attributed to it being a free radical scavenger and its ability to promote activity of various antioxidant enzymes, depending on the concentration of resveratrol and the target cell types, resveratrol can also exhibit pro-oxidant properties (de la Lastra and Villegas, 2007). In fact, recent studies indicated that resveratrol did not result in generation of ROS in E. coli (Subramanian et al., 2014; Haranahalli et al., 2016); the study instead suggested that the inhibition of $E$. coli by resveratrol may be mediated through sitespecific oxidative membrane damage (Subramanian et al., 2014) though the mechanism behind the membrane damage is still under investigation.

Another proposed antibacterial mechanism of resveratrol may be through the generation of $\mathrm{Cu}(\mathrm{II})$-peroxide complex upon reduction of $\mathrm{Cu}(\mathrm{II})$ to $\mathrm{Cu}(\mathrm{I})$ with resultant DNA cleavage and cell death (Fukuhara and Miyata, 1998). Fukuhara and Miyata (Fukuhara and Miyata, 1998) proposed that resveratrol binds to $\mathrm{Cu}(\mathrm{II})$ to form a $\mathrm{Cu}(\mathrm{II})$-peroxide complex, which leads to reduction of $\mathrm{Cu}(\mathrm{II})$ to $\mathrm{Cu}(\mathrm{I})$. The $\mathrm{Cu}(\mathrm{II})$-peroxide complex binds DNA to form DNA-resveratrol-Cu(II) ternary complex and this leads to DNA scission and breakage (Figure 3). The reduction of $\mathrm{Cu}(\mathrm{II})$ to $\mathrm{Cu}(\mathrm{I})$ is necessary for DNA cleavage to occur. When buthacuprione, a $\mathrm{Cu}(\mathrm{I})$ chelating agent, was added to solution containing resveratrol and $\mathrm{Cu}(\mathrm{II})$, there was a reduction in the levels of the nicked form of DNA produced from supercoiled DNA, indicating reduction in DNA cleavage (Fukuhara and Miyata, 1998). Additionally, the number and positions of the hydroxyl group in the stilbene backbone also have an effect on DNA cleavage activity. Resveratrol with a 4-hydroxy group has pro-oxidant activity but isoresveratrol (Figure 4A), an analog of resveratrol with a 3-hydroxy group did not reduce $\mathrm{Cu}(\mathrm{II})$ and cause DNA cleavage. The 4-hydroxy group is responsible for its high affinity binding to both $\mathrm{Cu}(\mathrm{II})$ and DNA in forming a ternary complex to cleave DNA efficiently. Furthermore, dihydroresveratrol (Figure 4B) was shown to have decreased DNA cleaving ability compared to resveratrol, which indicates the importance of the double bond in resveratrol which not only provides planarity to the structure for effective DNA binding, but also confers the stability of the 4-oxy radical form (Fukuhara et al., 2006). A more recent study by Subramanian et al. (2016) suggested that DNA damage induced by resveratrol in E. coli could be a much later event occurring only after the membrane integrity has been compromised, indicating that there is much to be explored in order to fully understand the mechanism of reservatrol's action.

Another proposed mechanism of resveratrol's antibacterial activity is through the inhibition of the electron transport chain (ETC) and F0F1-ATPase, thereby inhibiting the proliferation of pathogenic microorganisms by reducing cellular energy production (Madrigal-Perez and Ramos-Gomez, 2016). A study by Dadi et al. (2009) found that resveratrol inhibited E. coli ATP synthase activity, leaving only $60 \%$ residual activity. Resveratrol (at $100 \mu \mathrm{M}$ ) was also found to decrease complex III activity by competing with coenzyme Q, leading to about $20 \%$ reduction in enzyme activity (Zini et al., 1999). In another study, resveratrol was found to inhibit enzymatic activity of F0F1-ATPase/ATP synthase in rat brain and liver in a concentration-dependent manner (Zheng and Ramirez, 2000). Similarly, a significant reduction of metabolic activity was observed in the genus Arcobacter bacteria upon treatment with resveratrol (Ferreira et al., 2014).

Reservatrol also appears to have an inhibitory action on cell division through its actionon filamentous temperature sensitive protein (FtsZ), a GTP-dependent prokaryotic cell division protein responsible for cell division through formation of dynamic Z-ring in the middle of cell. Inhibiting expression of FtsZ gene leads to a decrease in Z-ring formation in cells and therefore inhibition of cell division (Haranahalli et al., 2016). By using phase contrast microscopy, Hwang and Lim (2015) observed that the length of resveratrol treated E. coli cells increased in a dose-dependent manner, suggesting that the elongation was due to interference of septum formation, which therefore indicated a failure of cell division. To confirm the effect of resveratrol on Z-ring formation, they used an E. coli strain JW0093 containing plasmid that expressed one or more Z-rings. Confocal microscopy showed that E. coli expressed Zring formation in the absence of resveratrol while treatment with resveratrol diminished the appearance of Z-rings. Western blot analysis was used to further confirm the inhibition of FtsZ expression by resveratrol, the analysis of which showed a decrease in FtsZ levels in E. coli treated with resveratrol. These results led to them conclude that the antibacterial activity of resveratrol was attributed to suppression of FtsZ gene expression, thereby inhibiting Z-ring formation and cell division (Hwang and Lim, 2015).

\section{ANTIBIOFILM AND ANTIVIRULENCE ACTIVITIES OF RESVERATROL AGAINST FOODBORNE BACTERIA}

One of bacteria's survival strategies is the ability to form biofilms where they adhere to various surfaces and form sessile biofilm communities using a self-produced extracellular polymeric matrix. These biofilms produced by pathogenic foodborne bacteria can cause serious health risks due to their inherent resistance to antimicrobial agents, host defenses and external stresses, which make them difficult to eradicate and can be an additional factor leading to antibiotic resistance (Cho et al., 2013, 2015; Lee J. H. et al., 2014). In food industries, the biofilms of spoilage or pathogenic bacteria represent a considerable problem as they form on food surfaces as well as the processing environment, leading to postprocessing contamination and cross-contamination. As an intercellular communication mechanism, quorum sensing regulates a variety of physiological functions of bacteria including the virulence and biofilm formation (Deep et al., 2011) which could limit bacterial 


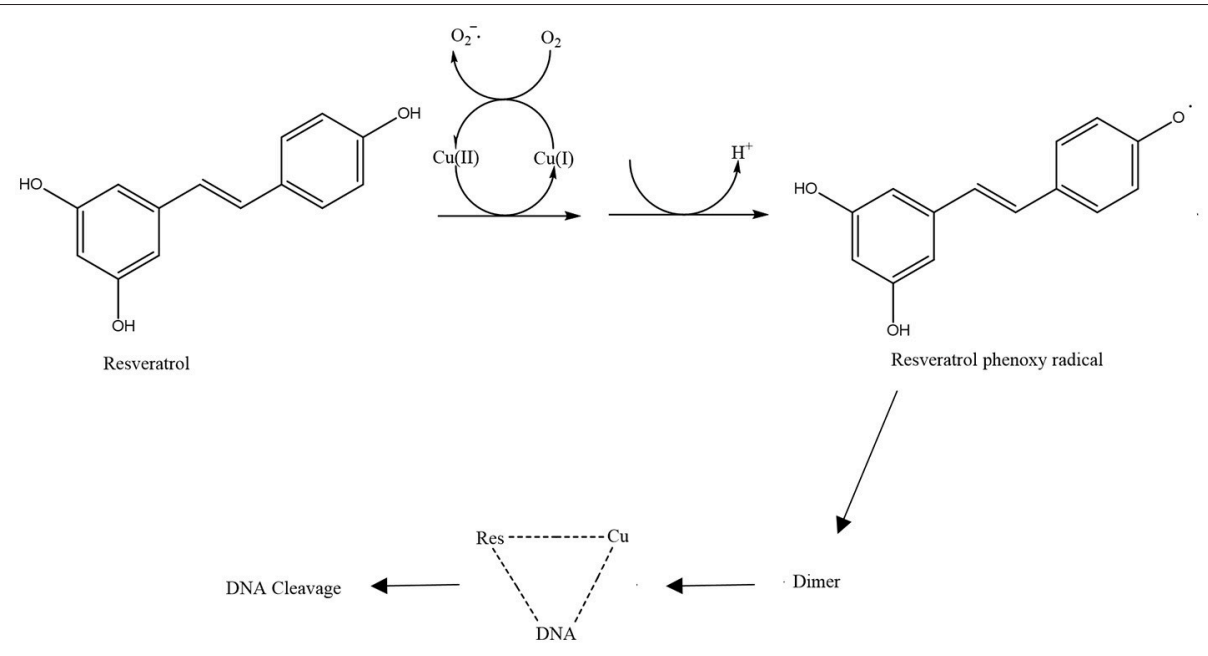

FIGURE 3 | Schematic diagram of resveratrol pro-oxidant activity (de la Lastra and Villegas, 2007).

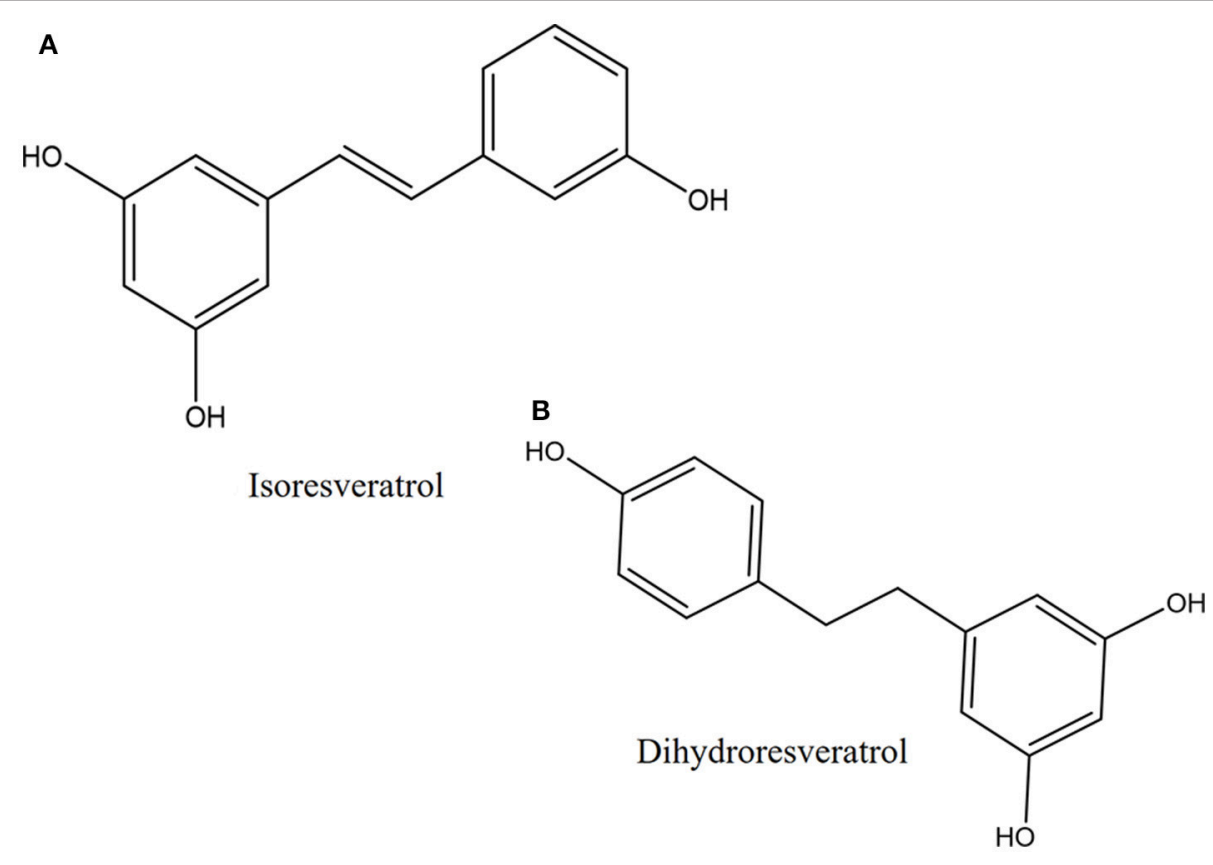

FIGURE 4 | Chemical structures of (A) isoresveratrol and (B) dihydroresveratrol.

proliferation in foods and food spoilage. Hence, new alternatives to antibiotics that could eradicate bacterial biofilms and diminish virulence of foodborne bacteria could be a promising strategy to ensure food safety and quality (Cho et al., 2013).

Considering the complexity and heterogeneity of biofilm structure, several parameters have to be taken into account when selecting the proper measurement endpoint for antibiofilm screens (Fallarero et al., 2014), which includes the measurement of the biomass, biofilm viability as well as the extracellular matrix of the biofilm. The literature documents several methods that have been employed to assess the antibiofilm activity of resveratrol; these methods include the quantification of the biofilm using crystal-violet dye (Lee K. et al., 2014; Qin et al., 2014), quantification of bacterial viability using metabolic assays (MTT) (Duarte et al., 2015) and microscopic evaluation using scanning electron microscope and confocal laser scanning microscope (Lee J. H. et al., 2014; Cho et al., 2015). Furthermore, studies also further investigated the antibiofilm associated mechanisms of resveratrol by assessing the effect of resveratrol on the genes associated to the regulation of biofilm development (Qin et al., 2014). Several studies have reported the biofilm inhibitory activity of resveratrol against both Gram 
positive and Gram-negative foodborne pathogens (Tables 3, 4). Differential activities of resveratrol in inhibiting biofilm formation and eradicating biofilm already formed were observed against specific species of foodborne pathogens. Previous work demonstrated that resveratrol appears effective in inhibiting biofilm formation by S. aureus (Moran et al., 2014; Qin et al., 2014), L. monocytogenes (Ferreira and Domingues, 2016), E. coli O157:H7 (Lee et al., 2013), Campylobacter sp. (Duarte et al., 2015), and V. cholerae (Augustine et al., 2014).

Qin et al. (2014) demonstrated that resveratrol at $100 \mu \mathrm{g} / \mathrm{mL}$ caused $39.85 \%$ inhibition of methicillin-resistant S. aureus (MRSA) biofilm formation and at $150 \mu \mathrm{g} / \mathrm{mL}$, caused $23.42 \%$ removal of preformed biofilm. This was also visualized by SEM where $100 \mu \mathrm{g} / \mathrm{mL}$ and $150 \mu \mathrm{g} / \mathrm{mL}$ resveratrol both resulted in thinner biofilm post-treatment. In addition, the combination of resveratrol $150 \mu \mathrm{g} / \mathrm{mL}$ and vancomycin $8 \mu \mathrm{g} / \mathrm{mL}$ resulted in $55.43 \%$ removal of preformed biofilm which suggested potential of resveratrol as adjunct therapy in MRSA biofilm infection for its synergistic activity (Qin et al., 2014). It was proposed the antibiofilm activity of resveratrol might be due to its ability to cause interference in quorum sensing thus affecting synthesis of surface proteins and capsular polysaccharidesdownregulation of genes (cap5ABCFG) responsible for capsular polysaccharide synthesis was reported with resveratrol treatment at $100 \mu \mathrm{g} / \mathrm{mL}$. The combination of resveratrol and vancomycin was also found to downregulate the virulence gene clfA and biofilm gene spoVG. The authors proposed that the synergistic activity of the resveratrol/vancomycin combination was due to vancomycin induced upregulation of the genes associated with purine metabolism (purADHN) which was observed in the combination treatment but not in resveratrol alone (Qin et al., 2014). As such, the combinatorial treatment was more effective in removing preformed biofilm as it induced purine metabolism and reduced capsular polysaccharide synthesis (Qin et al., 2014). Furthermore, there was additional evidence showing the inhibitory effect of resveratrol on biofilm formation by enteropathogenic $S$. aureus in which resveratrol at sub-inhibitory concentrations of $100 \mu \mathrm{g} / \mathrm{mL}$ exerted 20 to $45 \%$ antibiofilm activity (Moran et al., 2014).

However, there have been also been several previous studies reporting negative inhibitory effect of resveratrol against biofilm formation by methicillin-sensitive S. aureus (MSSA) (Lee K. et al., 2014; Cho et al., 2015). Lee K. et al. (2014) reported transresveratrol at $100 \mu \mathrm{g} / \mathrm{mL}$ had no significant effect in reducing biofilm formation in S. aureus ATCC6538 when compared to control. Similarly, Cho et al. (2015) also reported transresveratrol had no antibiofilm activity on S. aureus ATCC6538 at concentrations up to $100 \mu \mathrm{g} / \mathrm{mL}$. This data seems to highlight that the antibiofilm activity of resveratrol could be strain specific, particularly toward MRSA and enteropathogenic S. aureus but not $S$. aureus ATCC6538. The differential antibiofilm activity of resveratrol toward the different strains of $S$. aureus could be explained by the difference in the involvement of multiple molecules and factors including the adhesion factors, quorum sensing and capsular polymers possessed by the bacterial strains to form biofilm (Karatan and Watnick, 2009; Moran et al., 2014). Besides that, the divergent findings could also be related to the varying methodologies employed by respective studies in assessing the antibiofilm properties of resveratrol. Particularly, the spectroscopic assay using the crystal violet stain to quantify the total biofilm biomass is subject to several limitations, including species dependence, requirement for individual optimization of protocols (e.g., period for biofilm formation, development, maturation, staining) and it is also unable to discriminate between the different components in the biofilm (e.g., living and non-living cells; Pantanella et al., 2013; Bueno, 2014). To differentiate living or dead microorganisms within the biofilm, the crystal violet method could be coupled with bacterial viability assays such as conventional viable plate counting and metabolic assays such as MTT (Fallarero et al., 2014).

Contamination of food by L. monocytogenes is common in food processing environments due to their hardy growth characteristics and ability to attach to stainless steel and many surfaces to form biofilm (Donnelly, 2001). Often, $L$. monocytogenes is associated with foodborne disease outbreaks characterized by widespread distribution and high mortality rates. Thus, effective strategies to remove or inhibit biofilm formation by L. monocytogenes would be of tremendous value to the food processing industry. In 2016, Ferreira and Domingues (2016) demonstrated that resveratrol at MIC of $200 \mu \mathrm{g} / \mathrm{mL}$ produced approximately 60 to $70 \%$ biofilm inhibition against three L. monocytogenes strains (LMG 16779 serovar 1/2a, LMG 16780 serovar 1/2b, LMG 13305 serovar 4b) and Listeria innocua LOP 9 isolated from sheep's milk. They also reported that $L$. monocytogenes serovar $1 / 2 \mathrm{a}$, the most commonly found serovar in food processing plants was the most sensitive strain toward biofilm inhibition by resveratrol (Ferreira and Domingues, 2016). This study claimed to be the first to demonstrate the inhibitory effect of resveratrol against biofilm formation by $L$. monocytogenes (Ferreira and Domingues, 2016).

Trans-resveratrol was also shown to be an effective antibiofilm and antiquorum sensing agent against enterohemorrhagic E. coli O157:H7 (EHEC) (Lee et al., 2013). Using trans-resveratrol at $10 \mu \mathrm{g} / \mathrm{mL}$, biofilm formation as well as swimming and swarming abilities of EHEC were inhibited through down-regulation of several quorum sensing genes. They showed that resveratrol at $20 \mu \mathrm{g} / \mathrm{mL}$ downregulated the expression of curli genes ( $\operatorname{csg} A$ and $\operatorname{csg} B)$, motility gene $(f h D, f i m A, f i m H$, and $\operatorname{mot} B)$ and autoinducer-2 (AI-2) quorum sensing gene (lsrA, luxS, and luxR) (Lee et al., 2013). The study also demonstrated specific and selective inhibitory effect of trans-resveratrol against EHEC but did not harm the commensal E. coli strains, suggesting that possible application of resveratrol as an antibiofilm compound in combination with lower dose of antibiotics to maximize removal of pathogen without rendering development of antibiotic resistance while protecting commensal microflora (Lee et al., 2013).

C. jejuni and C. coli are the leading agents responsible for bacterial gastroenteritis in humans worldwide from consumption of contaminated poultry meat and products. They also form biofilms that are resistant to disinfectant and represent a major issue within the food industry. Several studies have been conducted to investigate the potential of resveratrol as an 


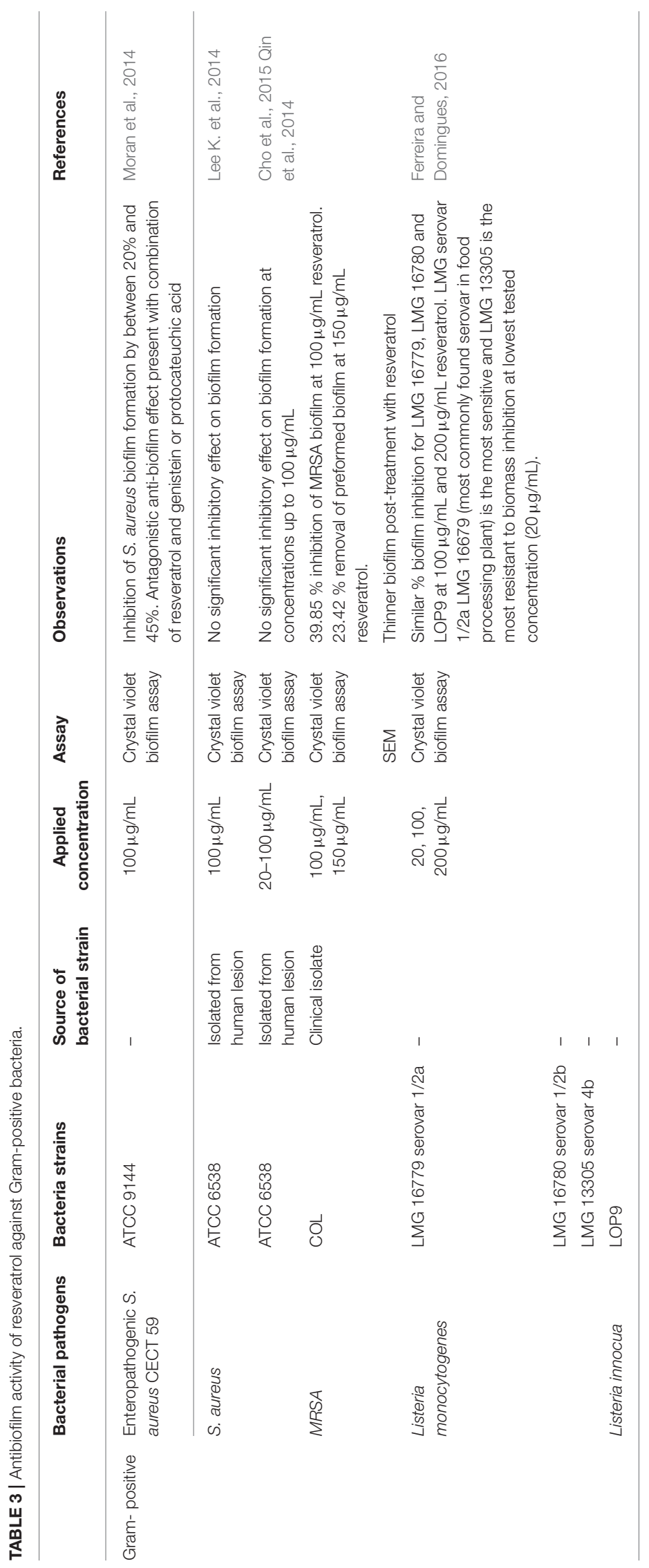




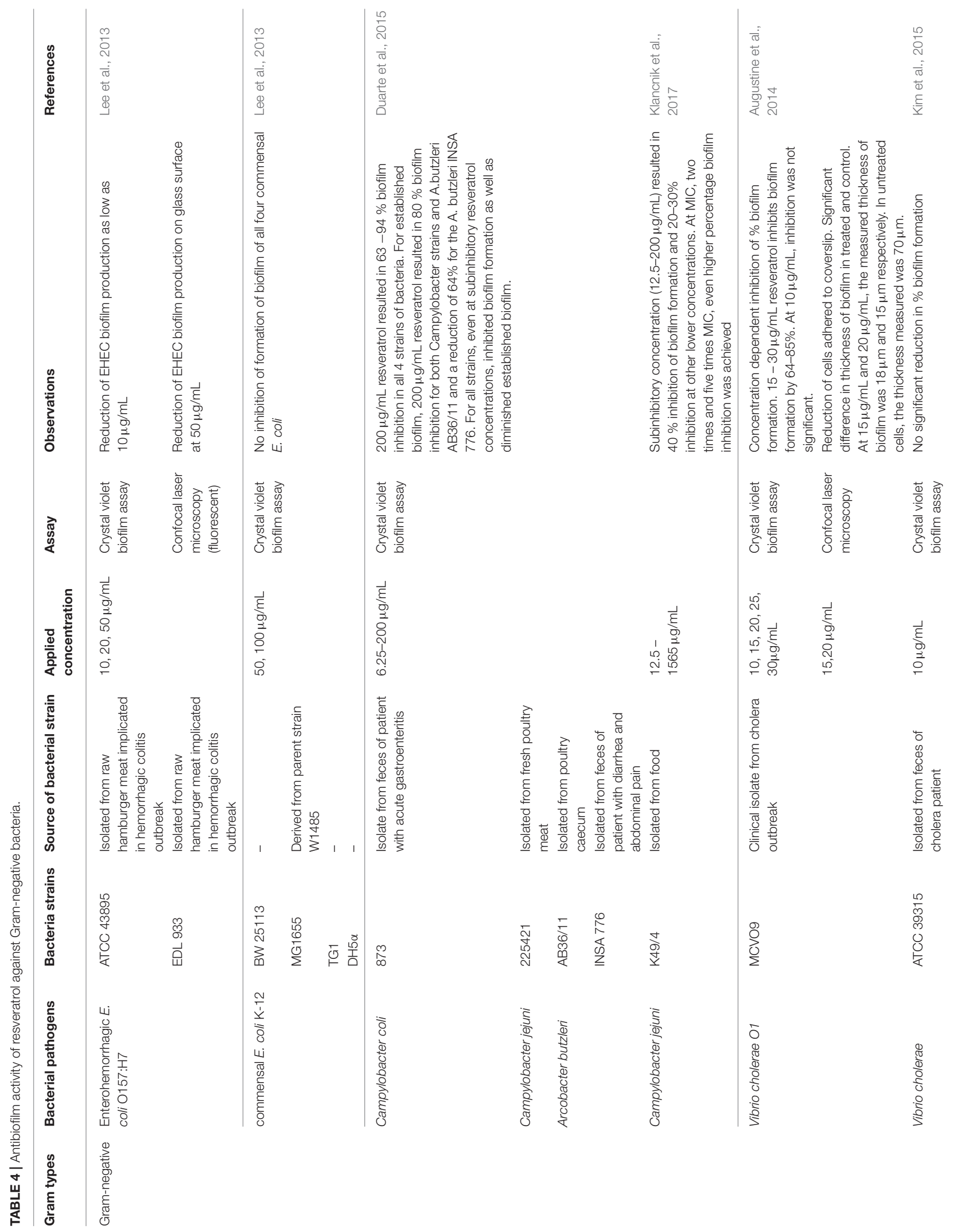


antibiofilm and antiquorum sensing agent against Campylobacter bacteria. Duarte et al. (2015) reported resveratrol at $200 \mu \mathrm{g} / \mathrm{mL}$ resulted in $63-93 \%$ biofilm inhibition in four tested bacteria strains (C. coli 873, C. jejuni 225421, and A. butzleri AB36/11 and INSA 776). With reference to preformed biofilm, $200 \mathrm{n} \mu \mathrm{g} / \mathrm{mL}$ resveratrol caused $80 \%$ reduction in biofilm formation for both Campylobacter strains and A. butzleri AM36/11, and 64\% reduction for A. butzleri INSA 776. The reported concentrationdependent antibiofilm activity of resveratrol in inhibiting biofilm formation and in diminishing preformed biofilm in all four bacteria strains even at sub-inhibitory concentration of 6.25$50 \mu \mathrm{g} / \mathrm{mL}$ suggests that there could be significant applications for this compound. The authors proposed that the effect of resveratrol on biofilm formation is related to interference in the expression of biofilm-related genes as shown in study by Lee et al. (2013), and the antibiofilm activity on preformed biofilm was related to the effect on quorum sensing (Reeser et al., 2007; Gölz et al., 2012). Klancnik et al. (2017) examined the effect of resveratrol on the inhibition of biofilm formation (measured as adhered biomass) by C. jejuni and the culturability and viability of the adhered cells post treatment with resveratrol. Sub-inhibitory concentration $(12.5-200 \mu \mathrm{g} / \mathrm{mL})$ of resveratrol resulted in $40 \%$ inhibition of $C$. jejuni biofilm formation and $20-$ $30 \%$ inhibition was achieved at other lower concentrations tested. When tested at MIC, $2 \mathrm{x}$ MIC and $5 \mathrm{x}$ MIC $(\mathrm{MIC}=313 \mu \mathrm{g} / \mathrm{mL})$, resveratrol resulted in even higher percentage inhibition in biomass formation, and reduced culturability and viability of the adhered cells by $50-60 \%$.

Resveratrol was also shown to exhibit antibiofilm activity against Vibrio bacteria. Augustine et al. (2014) reported concentration dependent inhibition of percentage biofilm formation in Vibrio cholerae O1 MCVO9; at $15-30 \mu \mathrm{g} / \mathrm{mL}$, resveratrol inhibited biofilm formation by $64-85 \%$. Reduction of cells adherent to the coverslip was visualized using confocal laser microscopy whereby the thickness of the biofilm formed was significantly different between resveratrol treated cells and control. Meanwhile, Kim et al. (2015) reported no significant reduction in percentage biofilm formation in Vibrio cholera O1 El Tor serotype Inada strain N16961 after treated with $10 \mu \mathrm{g} / \mathrm{mL}$ of resveratrol. Perhaps, a higher concentration of resveratrol is needed to inhibit the biofilm formation by $V$. cholera O1 El Tor serotype as shown by the earlier study with positive inhibitory effect by resveratrol at $15-30 \mu \mathrm{g} / \mathrm{mL}$ against V. cholerae O1 MCVO9 (Augustine et al., 2014). Besides the selective antibiofilm activity demonstrated by resveratrol against $V$. cholerae, it was demonstrated that resveratrol inhibited cholera toxin induced cAMP accumulation in Vero cells through the suppression of cholera toxin internalization (Morinaga et al., 2010). Collectively, the evidence suggests that resveratrol has the potential to be developed into an antiVibrio agent that provides protective effects against cholera infection.

Although the existing evidence indicates that resveratrol exhibits antibiofilm properties against various foodborne pathogens, substantial efforts using reliable quantification methods are still needed in order to show that resveratrol is truly an effective antibiofilm agent. Clearly, the current evidence warrants further work to elucidate the exact mechanism of action of resveratrol in the disruption or inhibition of foodborne pathogen biofilms. Also, these antibiofilm properties of resveratrol hold promise for the development of a molecule that may be used either on its own or as an adjuvant to existing suppression or eradication strategies against foodborne pathogens.

\section{APPLICATION AND EFFICACY OF RESVERATROL IN THE FOOD SYSTEM}

While the laboratory results are very encouraging, it should be borne in mind that there are potential limitations to the degree to which the study findings may be applied in real world situations. Food and food products are rich in complex nutrients, which may support growth of bacteria even better than nutrient media, and it is likely a higher concentration of antimicrobial might be needed to achieve the desired results even though substantial antimicrobial activity is achieved in invitro studies (Shelef, 1984; Gill et al., 2002). Intrinsic properties such as fat, protein, water content, antioxidant, $\mathrm{pH}$, food additive and extrinsic properties such as storage condition, temperature, and microorganism characteristics can also potentially diminish the efficacy of the antibacterial agent (Tassou et al., 1995). Furthermore, the possibility of nutritional and organoleptic losses should also be noted when using an antibacterial agent to improve microbial quality and safety of food. For example, in the case of essential oils, despite their greater antimicrobial potential (Burt, 2004; Lai et al., 2017) than resveratrol, their application in food preservation is still limited by their intense aroma and toxicity issues.

For resveratrol, even though promising results were obtained from the in vitro experiments including the agar and broth systems, applied studies are also mandatory to confirm its effectiveness in food protection against foodborne pathogens. Surendran Nair et al. (2016) examined the enhancement of thermal inactivation of E. coli O157:H7 inoculated on beef patties in the presence of resveratrol. They reported no decline in $E$. coli count irrespective of treatment throughout the refrigerated storage ( $4^{\circ} \mathrm{C}$ for 5 days) in uncooked patties. In patties that are stored at $4^{\circ} \mathrm{C}$ for 5 days and cooked to an internal temperature of $65^{\circ} \mathrm{C}$, significant reduction ( $>3 \log \mathrm{CFU} / \mathrm{g}$ ) of E. coli was observed for day 1,3 , and 5 at $0.2 \% \mathrm{w} / \mathrm{w}$ resveratrol in comparison to control whereas at $0.1 \% \mathrm{w} / \mathrm{w}$, significant reduction was observed at day 3 and 5. A synergistic effect was observed when either concentration of resveratrol was combined with chitosan $(1 \% \mathrm{w} / \mathrm{w})$, which significantly reduced count, while chitosan + resveratrol $0.2 \% \mathrm{w} / \mathrm{w}$ completely diminished E. coli count in day 3 and 5 (Surendran Nair et al., 2016). They proposed the synergistic effect observed was because chitosan, being an emulsifier, can solubilise resveratrol, thereby improving the distribution of resveratrol in meat (Klinkesorn, 2013). Enhancement of thermal activation in the presence of resveratrol suggested that heat caused damage to cell membrane integrity, thereby allowing better penetration of resveratrol into cells. Similarly, resveratrol was shown to cause oxidative damage to 
cell membrane, which may help to enhance the killing when compared to heat alone (Subramanian et al., 2014).

Ferreira and Domingues (2016) demonstrated the antibacterial effect of resveratrol $(200-400 \mu \mathrm{g} / \mathrm{mL})$ against L. monocytogenes and L. innocua in four food models: UHT treated skim milk, UHT treated whole milk, chicken juice and lettuce leaf. They reported resveratrol significantly reduced bacterial count for both strains in chicken juice and lettuce leaf model. The effect was less pronounced for L. monocytogenes in UHT-treated skim and whole milk, with no significant reduction of bacteria in the whole milk. They proposed the observed difference was due to higher fat content in whole milk which protected the bacteria from inhibition by resveratrol. Due to the hydrophobic nature of resveratrol, it might preferentially dissolve in the lipid phase of milk, thus decreasing the amount of active substance available to act on bacteria (Mejlholm and Dalgaard, 2002).

Consumption of raw vegetables also represents a common source of food borne infections, thus it is critical to control the microbial load in the fresh produce particularly from the salad supply chain, ensuring food safety and hygiene quality. Lai et al. (2017) demonstrated the potential of resveratrol to be used as a natural preservative to control the total viable microbial load in alfalfa sprout and mushroom slices. They reported $0.9 \mathrm{log}$ reduction and $3.06 \log$ reduction in microbial load in alfalfa sprout and mushroom respectively after $24 \mathrm{~h}$ storage at $4^{\circ} \mathrm{C}$ (Lai et al., 2017).

There are some concerns with reference to resveratrol's impact on the organoleptic properties of the food, as it may impart an astringent flavor and bitterness to the food. Gaudette and Pickering (2011) reported an increase in the perception of bitterness with increasing concentrations of resveratrol added to wine. To minimize the effect of resveratrol on the organoleptic properties of the food, techniques such as encapsulation of resveratrol by spray-drying was suggested by Koga et al. (2015) to reduce the perception of bitterness. They reported a higher taste detection threshold for resveratrol $(313 \mu \mathrm{g} / \mathrm{mL})$ encapsulated with sodium caseinate than unencapsulated resveratrol $(90 \mu \mathrm{g} / \mathrm{mL})$ in protein solution (Koga et al., 2015). To date, studies on the sensory evaluation of resveratrol in the food system are still limited, thus further work is needed to improve the utilization of resveratrol as a food preservatives by minimizing its taste altering effect.

\section{BIOAVAILABILITY, SAFETY PROFILE, AND DRUG-DRUG INTERACTIONS OF RESVERATROL}

Resveratrol was reported to be extensively absorbed and metabolized but with very low bioavailability. Walle et al. (2004) reported that $70 \%$ of an oral dose of $25 \mathrm{mg}$ resveratrol was absorbed but the concentration remaining unchanged in plasma was $<5 \mathrm{ng} / \mathrm{ml}$. They proposed that this was because resveratrol was metabolized in liver and intestine by sulfate and glucuronic acid conjugation and by hydrogenation of aliphatic double bond. In addition to that, inter-individual variability of resveratrol metabolism was also reported by Bode et al. (2013), where they proposed the difference seen might be attributed to variation in human gut microflora in microbial transformation of resveratrol. Thus, to effectively utilize resveratrol in foods and pharmaceuticals, many efforts have been reported to improve the bioavailability of resveratrol through various encapsulation methods.

The safety profile of resveratrol has been well documented and is safe for consumption up to $5 \mathrm{~g}$ per day in healthy humans. Several studies reported that gastrointestinal side effects such as diarrhea, nausea, and abdominal pain were common when administered at high doses (Brown et al., 2010; Chow et al., 2010; Howells et al., 2011; Anton et al., 2014; Sergides et al., 2016). Brown et al. (2010) reported mild gastrointestinal symptoms when resveratrol was administered at a doses of $2.5 \mathrm{~g} /$ day while Sergides et al. (2016) reported resveratrol administered 500 $\mathrm{mg} /$ day was well tolerated in human. Similarly, Howells et al. (2011) reported mild gastrointestinal symptoms as the primary side effect experienced by patients taking resveratrol $5 \mathrm{~g} /$ day, with other less common side effects such as chills, rash, skin irritation and vascular flushing also reported. Anton et al. (2014) reported short-term resveratrol administration (90 days) at $300 \mathrm{mg} /$ day and $1 \mathrm{~g} /$ day was well tolerated in overweight elderly and did not significantly altered blood chemistries. Chow et al. (2010) also reported high tolerability of resveratrol administered at $1 \mathrm{~g} /$ day as most side effects were mild and transient.

The potential drug-resveratrol interaction is also an important aspect requiring considerable attention. Co-administration of resveratrol together with other drugs may potentially result in drug-resveratrol interactions by changing the pharmacokinetics of the drug or resveratrol. Resveratrol inhibits several key CYP enzymes such as CYP3A4, 1A2, 2C9, 2D6, and 2E1. Thus, high intake of resveratrol may theoretically reduce the metabolism of drugs by CYP enzymes and increase their bioavailability and potential toxicity. In a clinical trial study by Chow et al. (2010), they reported 1.33-fold increase in AUC for buspirone following co-administration of resveratrol at $1 \mathrm{~g}$ /day for 4 weeks due to inhibition of CYP3A4. Similarly, Choi et al. (2009) reported 2.2 and 2.3-fold increase in $C_{\max }$ and AUC of nicardipine respectively in male rats following co-administration of resveratrol at $10 \mathrm{mg} / \mathrm{kg}$ body weight. Potential systemic drugresveratrol interaction should be considered at high doses at $>1 \mathrm{~g} /$ day. However, the risk of interaction is minimal at low milligram doses as resveratrol has a very low bioavailability and is rapidly metabolized by conjugation in vivo. Therefore, the systemic concentration of free resveratrol is far below the conjugates. In addition, there have been no reports so far of inhibition of CYPs by resveratrol conjugates, and high plasma protein binding of resveratrol also reduces the risk of potential interactions (Detampel et al., 2012).

\section{COMMERCIALIZATION AND FUTURE DIRECTIONS}

At the time of writing, more than 5000 patents were found from searching "Scopus" using search terms 
"resveratrol" AND “antibacterial" OR "antimicrobial” limit to title/abstract/keywords. Some of the patented products containing resveratrol included antibacterial foot spray and hand cleanser (Yoo and Kim, 2013; Zhu et al., 2016), botanical fungicide (Li et al., 2015), skin care products (He et al., 2017; Lei et al., 2017), food supplements (Sardi and Solomonson, 2005; Pall, 2014; Arroyo Paz, 2017) and antioxidant eye drops (Kador, 2016). Clearly, in the future, more research is required to unravel the potential of resveratrol to be developed into biopharmaceutical products. As the studies currently focus only on in-vitro determination of biological activity of resveratrol, there is an urgent need for studies on the efficacy of resveratrol in in vivo animal models associated with foodborne infections. In addition, to obtain the full potential of resveratrol, further evaluation of the mechanism of action of resveratrol will be needed to strengthen its practical applications and significance in future research. Further investigations could be extended to interactions between resveratrol and the food ingredients or food additives as well as its effect on the organoleptic properties of the product. Studies of the stability of resveratrol under various storage conditions and processing conditions are also needed to confirm its applicability in the food industry.

\section{CONCLUSION}

Food contamination by pathogenic foodborne bacteria is a major health risk to the public and the situation is being further exacerbated by the emergence of antibiotic resistant strains which are promoted by the ever-increasing usage of antibiotics. Natural antimicrobials seem to hold promise as an alternative to antibiotics and can be used to decrease the spread of resistant strains. Resveratrol exhibits promising antibacterial activities against important foodborne bacteria including $S$. aureus, $L$. monocytogenes, C. jejuni, E. coli, and V. cholerae, possibly

\section{REFERENCES}

Alvarez, M. V., Moreira, M. R., and Ponce, A. (2012). Antiquorum sensing and antimicrobial activity of natural agents with potential use in food. J. Food Saf. 32, 379-387. doi: 10.1111/j.1745-4565.2012.00390.x

Alvarez, M. V., Ponce, A. G., and Moreira, M. D. R. (2013). Antimicrobial efficiency of chitosan coating enriched with bioactive compounds to improve the safety of fresh cut broccoli. LWT Food Sci. Technol. 50, 78-87. doi: 10.1016/j.lwt.2012.06.021

Anton, S. D., Embry, C., Marsiske, M., Lu, X., Doss, H., Leeuwenburgh, C., et al. (2014). Safety and metabolic outcomes of resveratrol supplementation in older adults: results of a twelve-week, placebo-controlled pilot study. Exp. Gerontol. 57, 181-187. doi: 10.1016/j.exger.2014.05.015

Arroyo Paz, F. E. (2017). Anti-aging Supplement. MX 2016002359 A 20170822. Mexico: Patent and Trademark Office.

Augustine, N., Goel, A. K., Sivakumar, K. C., Kumar, R. A., and Thomas, S. (2014). Resveratrol-a potential inhibitor of biofilm formation in Vibrio cholerae. Phytomedicine 21, 286-289. doi: 10.1016/j.phymed.2013.09.010

Bavaresco, L., Vezzulli, S., Civardi, S., Gatti, M., Battilani, P., Pietri, A., et al. (2008). Effect of lime-induced leaf chlorosis on ochratoxin A, trans-resveratrol, and epsilon-viniferin production in grapevine (Vitis vinifera L.) berries infected by Aspergillus carbonarius. J. Agric. Food Chem. 56, 2085-2089. doi: $10.1021 /$ jf073456+ mediated through DNA cleavage, membrane damage, reduced cellular metabolic activity and inhibition of cell division. The antibiofilm and antivirulence activities of resveratrol can be exploited as stand-alone alternative therapy or as coadjuvants to current antibiotic therapy against foodborne pathogens. Furthermore, resveratrol also proved to be effective in inhibiting several foodborne pathogens in different types of food matrix, which supports its potential use as an alternative food additive or preservative to ensure high quality food products which are safe to be consumed. Based on the current toxicological data, resveratrol was shown have low toxicity even when taken at high doses with mild gastrointestinal disturbances being the major side effect reported. Overall, this review highlights the potential application of resveratrol as an antimicrobial biopharmaceutical product as well as to have significant applications in the food industry as a food preservative.

\section{AUTHORS CONTRIBUTIONS}

The writing was performed by DM, LT-HT, PP, L-HL, and B-HG. While WY, LH-C, TK, K-GC, LM, L-HL, and B-HG provided vital guidance and insight to the work. The project was conceptualized by L-HL and B-HG.

\section{ACKNOWLEDGMENTS}

This work was inspired by Monash Pharmacy Degree Course, Unit PAC3512 which entitled "Current aspects of pharmaceutical research" and financially supported by the Monash University Malaysia ECR Grant (5140077-000-00), MOSTI eScience Fund (02-02-10-SF0215), University grants from the University of Malaya (GA001-2016, GA002-2016, PG226-2016A to K-GC) and External Industry Grants from Biotek Abadi Sdn Bhd (vote no. GBA-808138 and GBA-808813).

Beveridge, T. J. (1999). Structures of gram-negative cell walls and their derived membrane vesicles. J. Bacteriol. 181, 4725-4733.

Bode, L. M., Bunzel, D., Huch, M., Cho, G. S., Ruhland, D., Bunzel, M., et al. (2013). In vivo and in vitro metabolism of trans-resveratrol by human gut microbiota. Am. J. Clin. Nutr. 97, 295-309. doi: 10.3945/ajcn.112.049379

Bombardelli, E., and Morazzoni, P. (1995). Vitis vinifera L. Fitoterapia 66, 291-317. Brown, V. A., Patel, K. R., Viskaduraki, M., Crowell, J. A., Perloff, M., Booth, T. D., et al. (2010). Repeat dose study of the cancer chemopreventive agent resveratrol in healthy volunteers: safety, pharmacokinetics, and effect on the insulin-like growth factor axis. Cancer Res. 70, 9003-9011. doi: 10.1158/0008-5472.CAN-10-2364

Bueno, J. (2014). Anti-biofilm drug susceptibility testing methods: looking for new strategies against resistance mechanism. J. Microbial Biochem. Technol. S3:004. doi: 10.4172/1948-5948.S3-004

Burt, S. (2004). Essential oils: their antibacterial properties and potential applications in foods-a review. Int. J. Food Microbiol. 94, 223-253. doi: 10.1016/j.ijfoodmicro.2004.03.022

Catalgol, B., Batirel, S., Taga, Y., and Ozer, N. K. (2012). Resveratrol: French paradox revisited. Front. Pharmacol. 3:141. doi: 10.3389/fphar.2012.00141

Chachay, V. S., Kirkpatrick, C. M., Hickman, I. J., Ferguson, M., Prins, J. B., and Martin, J. H. (2011). Resveratrol-pills to replace a healthy diet? Br. J. Clin. Pharmacol. 72, 27-38. doi: 10.1111/j.1365-2125.2011.0 3966.x 
Chan, M. M. (2002). Antimicrobial effect of resveratrol on dermatophytes and bacterial pathogens of the skin. Biochem. Pharmacol. 63, 99-104. doi: 10.1016/S0006-2952(01)00886-3

Chan, C. K., Tan, L. T. H., Andy, S. N., Kamarudin, M. N. A., Goh, B. H., and Kadir, H. A. (2017). Anti-neuroinflammatory activity of Elephantopus scaber L. via activation of Nrf2/HO-1 signaling and inhibition of p38 MAPK pathway in LPS-induced microglia BV-2 cells. Front. Pharmacol. 8:397. doi: 10.3389/fphar.2017.00397

Chan, W. K., Tan, L. T., Chan, K. G., Lee, L. H., and Goh, B. H. (2016). Nerolidol: a sesquiterpene alcohol with multi-faceted pharmacological and biological activities. Molecules 21:529. doi: 10.3390/molecules21050529

Cho, H. S., Lee, J. H., Cho, M. H., and Lee, J. (2015). Red wines and flavonoids diminish Staphylococcus aureus virulence with anti-biofilm and anti-hemolytic activities. Biofouling 31, 1-11. doi: 10.1080/08927014.2014.991319

Cho, H. S., Lee, J. H., Ryu, S. Y., Joo, S. W., Cho, M. H., and Lee, J. (2013). Inhibition of Pseudomonas aeruginosa and Escherichia coli O157:H7 biofilm formation by plant metabolite epsilon-viniferin. J. Agric. Food Chem. 61, 7120-7126. doi: $10.1021 / \mathrm{jf} 4009313$

Choi, J. S., Choi, B. C., and Kang, K. W. (2009). Effect of resveratrol on the pharmacokinetics of oral and intravenous nicardipine in rats: possible role of P-glycoprotein inhibition by resveratrol. Pharmazie 64, 49-52. doi: $10.1691 / \mathrm{ph} .2008 .8683$

Chow, H. H., Garland, L. L., Hsu, C. H., Vining, D. R., Chew, W. M., Miller, J. A., et al. (2010). Resveratrol modulates drug- and carcinogen-metabolizing enzymes in a healthy volunteer study. Cancer Prev. Res. 3, 1168-1175. doi: 10.1158/1940-6207.CAPR-09-0155

Dadi, P. K., Ahmad, M., and Ahmad, Z. (2009). Inhibition of ATPase activity of Escherichia coli ATP synthase by polyphenols. Int. J. Biol. Macromol. 45, 72-79. doi: 10.1016/j.ijbiomac.2009.04.004

Das, Q., Islam, M. R., Marcone, M. F., Warriner, K., and Diarra, M. S. (2017). Potential of berry extracts to control foodborne pathogens. Food Control 73, 650-662. doi: 10.1016/j.foodcont.2016.09.019

Deep, A., Chaudhary, U., and Gupta, V. (2011). Quorum sensing and bacterial pathogenicity: from molecules to disease. J. Lab. Physicians 3, 4-11. doi: 10.4103/0974-2727.78553

de la Lastra, C. A., and Villegas, I. (2007). Resveratrol as an antioxidant and prooxidant agent: mechanisms and clinical implications. Biochem. Soc. Trans. 35, 1156-1160. doi: 10.1042/BST0351156

Detampel, P., Beck, M., Krähenbühl, S., and Huwyler, J. (2012). Drug interaction potential of resveratrol. Drug Metab. Rev. 44, 253-265. doi: $10.3109 / 03602532.2012 .700715$

Donnelly, C. W. (2001). Listeria monocytogenes: a continuing challenge. Nutr. Rev. 59, 183-194. doi: 10.1111/j.1753-4887.2001.tb07011.x

Duarte, A., Alves, A. C., Ferreira, S., Silva, F., and Domingues, F. C. (2015). Resveratrol inclusion complexes: antibacterial and anti-biofilm activity against Campylobacter spp. and Arcobacter butzleri. Food Res. Int. 77, 244-250. doi: 10.1016/j.foodres.2015.05.047

Fabricant, D. S., and Farnsworth, N. R. (2001). The value of plants used in traditional medicine for drug discovery. Environ. Health Perspect. 109, 69-75. doi: 10.1289/ehp.01109s 169

Fallarero, A., Hanski, L., and Vuorela, P. (2014). How to translate a bioassay into a screening assay for natural products: general considerations and implementation of antimicrobial screens. Planta Med. 80, 1182-1199. doi: $10.1055 / \mathrm{s}-0034-1383061$

Fernández-Mar, M. I., Mateos, R., García-Parrilla, M. C., Puertas, B., and Cantos-Villar, E. (2012). Bioactive compounds in wine: resveratrol, hydroxytyrosol and melatonin: a review. Food Chem. 130, 797-813. doi: 10.1016/j.foodchem.2011.08.023

Ferreira, S., and Domingues, F. (2016). The antimicrobial action of resveratrol against Listeria monocytogenes in food-based models and its antibiofilm properties. J. Sci. Food Agric. 96, 4531-4535. doi: 10.1002/jsfa.7669

Ferreira, S., Silva, F., Queiroz, J. A., Oleastro, M., and Domingues, F. C. (2014). Resveratrol against Arcobacter butzleri and Arcobacter cryaerophilus: activity and effect on cellular functions. Int. J. Food Microbiol. 180, 62-68. doi: 10.1016/j.ijfoodmicro.2014.04.004

Filip, V., Plocková, M., Šmidrkal, J., Špičková, Z., Melzoch, K., and Schmidt, Š. (2003). Resveratrol and its antioxidant and antimicrobial effectiveness. Food Chem. 83, 585-593. doi: 10.1016/S0308-8146(03)00157-2
Fukuhara, K., and Miyata, N. (1998). Resveratrol as a new type of DNA-cleaving agent. Bioorg. Med. Chem. Lett. 8, 3187-3192. doi: 10.1016/S0960-894X(98)00585-X

Fukuhara, K., Nagakawa, M., Nakanishi, I., Ohkubo, K., Imai, K., Urano, S., et al. (2006). Structural basis for DNA-cleaving activity of resveratrol in the presence of $\mathrm{Cu}$ (II). Bioorg. Med. Chem. 14, 1437-1443. doi: 10.1016/j.bmc.2005.09.070

Gaudette, N. J., and Pickering, G. J. (2011). Sensory and chemical characteristics of trans-resveratrol-fortified wine. Aust. J. Grape Wine Res. 17, 249-257. doi: 10.1111/j.1755-0238.2011.00144.x

Gill, A. O., Delaquis, P., Russo, P., and Holley, R. A. (2002). Evaluation of antilisterial action of cilantro oil on vacuum packed ham. Int. J. Food Microbiol. 73, 83-92. doi: 10.1016/S0168-1605(01)00712-7

Gölz, G., Sharbati, S., Backert, S., and Alter, T. (2012). Quorum sensing dependent phenotypes and their molecular mechanisms in Campylobacterales. Eur. J. Microbiol. Immunol. 2, 50-60. doi: 10.1556/EuJMI.2.2012.1.8

Goswami, M., Mangoli, S. H., and Jawali, N. (2006). Involvement of reactive oxygen species in the action of ciprofloxacin against Escherichia coli. Antimicrob. Agents Chemother. 50, 949-954. doi: 10.1128/AAC.50.3.949-954.2006

Haranahalli, K., Tong, S., and Ojima, I. (2016). Recent advances in the discovery and development of antibacterial agents targeting the cell-division protein FtsZ. Bioorg. Med. Chem. 24, 6354-6369. doi: 10.1016/j.bmc.2016.05.003

He, T., Ke, Y., and Zou, Y. (2017). Cosmetic Composition Containing Nano Platinum Colloid and Resveratrol. CN 106924063 A 20170707. Faming Zhuanli Shenqing.

Howells, L. M., Berry, D. P., Elliott, P. J., Jacobson, E. W., Hoffmann, E., Hegarty, B., et al. (2011). Phase I randomized, double-blind pilot study of micronized resveratrol (SRT501) in patients with hepatic metastases-safety, pharmacokinetics, and pharmacodynamics. Cancer Prev. Res. 4, 1419-1425. doi: 10.1158/1940-6207.CAPR-11-0148

Hwang, D., and Lim, Y. H. (2015). Resveratrol antibacterial activity against Escherichia coli is mediated by Z-ring formation inhibition via suppression of FtsZ expression. Sci. Rep. 5:10029. doi: 10.1038/srep10029

Jang, M., Cai, L., Udeani, G. O., Slowing, K. V., Thomas, C. F., Beecher, C. W., et al. (1997). Cancer chemopreventive activity of resveratrol, a natural product derived from grapes. Science 275, 218-220. doi: 10.1126/science.275.5297.218

Jeandet, P., Bessis, R., Sbaghi, M., and Meunier, P. (1995). Production of the phytoalexin resveratrol by grapes as a response to Botrytis attack under natural conditions. J. Phytopathology 143, 135-139. doi: 10.1111/j.1439-0434.1995.tb00246.x

Jung, C. M., Heinze, T. M., Schnackenberg, L. K., Mullis, L. B., Elkins, S. A., Elkins, C. A., et al. (2009). Interaction of dietary resveratrol with animal-associated bacteria. FEMS Microbiol. Lett. 297, 266-273. doi: 10.1111/j.1574-6968.2009.01691.x

Jung, H. J., Hwang, I. A., Sung, W. S., Kang, H., Kang, B. S., Seu, Y. B., et al. (2005). Fungicidal effect of resveratrol on human infectious fungi. Arch. Pharm. Res. 28, 557-560. doi: 10.1007/BF02977758

Jung, H. J., Seu, Y. B., and Lee, D. G. (2007). Candicidal action of resveratrol isolated from grapes on human pathogenic yeast C. albicans. J. Microbiol. Biotechnol. 17, 1324-1329.

Kador, P. F. (2016). Antioxidant Eye Drops Comprising Nutraceuticals, with AntiInflammatory and Chelating Activity, in Carbomer Gel Carrier. American Chemical Society (ACS).

Karatan, E., and Watnick, P. (2009). Signals, regulatory networks, and materials that build and break bacterial biofilms. Microbiol. Mol. Biol. Rev. 73, 310-347. doi: 10.1128/MMBR.00041-08

Kim, H. I., Kim, J. A., Choi, E. J., Harris, J. B., Jeong, S. Y., Son, S. J., et al. (2015). In vitro and in vivo antimicrobial efficacy of natural plant-derived compounds against Vibrio cholerae of O1 El Tor Inaba serotype. Biosci. Biotechnol. Biochem. 79, 475-483. doi: 10.1080/09168451.2014.991685

Kirk, M. D., Pires, S. M., Black, R. E., Caipo, M., Crump, J. A., Devleesschauwer, B., et al. (2015). World Health Organization estimates of the global and regional disease burden of 22 foodborne bacterial, protozoal, and viral diseases, 2010: a data synthesis. PLoS Med. 12: e1001921. doi: 10.1371/journal.pmed.1001921

Klancnik, A., Sikic Pogacar, M., Trost, K., Tusek Znidaric, M., Mozetic Vodopivec, B., and Smole Mozina, S. (2017). Anti-campylobacter activity of resveratrol and an extract from waste Pinot noir grape skins and seeds, and resistance of Camp. jejuni planktonic and biofilm cells, mediated via the CmeABC efflux pump. $J$. Appl. Microbiol. 122, 65-77. doi: 10.1111/jam.13315 
Klinkesorn, U. (2013). The role of chitosan in emulsion formation and stabilization. Food Rev. Int. 29, 371-393. doi: 10.1080/87559129.2013.818013

Koga, C. C., Becraft, A. R., Lee, Y., and Lee, S. Y. (2015). Taste detection thresholds of resveratrol. J. Food Sci. 80, S2064-S2070. doi: 10.1111/1750-3841.12976

Kumar, S. N., Siji, J. V., Nambisan, B., and Mohandas, C. (2012a). Activity and synergistic interactions of stilbenes and antibiotic combinations against bacteria in vitro. World J. Microbiol. Biotechnol. 28, 3143-3150. doi: 10.1007/s11274-012-1124-0

Kumar, S. N., Siji, J. V., Rajasekharan, K. N., Nambisan, B., and Mohandas, C. (2012b). Bioactive stilbenes from a Bacillus sp. N strain associated with a novel rhabditid entomopathogenic nematode. Lett. Appl. Microbiol. 54, 410-417. doi: 10.1111/j.1472-765X.2012.03223.x

Lai, L. J., Chiu, J. M., and Chiou, R. Y. (2017). Fresh preservation of alfalfa sprouts and mushroom slices by soaking with thymol and resveratrol solutions. Food Sci. Nutr. 5, 776-783. doi: 10.1002/fsn3.458

Lee, J. H., Cho, H. S., Joo, S. W., Chandra Regmi, S., Kim, J. A., Ryu, C. M., et al. (2013). Diverse plant extracts and trans-resveratrol inhibit biofilm formation and swarming of Escherichia coli O157:H7. Biofouling 29, 1189-1203. doi: 10.1080/08927014.2013.832223

Lee, J. H., Kim, Y. G., Ryu, S. Y., Cho, M. H., and Lee, J. (2014). Resveratrol oligomers inhibit biofilm formation of Escherichia coli O157:H7 and Pseudomonas aeruginosa. J. Nat. Prod. 77, 168-172. doi: 10.1021/np400756g

Lee, K., Lee, J. H., Ryu, S. Y., Cho, M. H., and Lee, J. (2014). Stilbenes reduce Staphylococcus aureus hemolysis, biofilm formation, and virulence. Foodborne Pathog. Dis. 11, 710-717. doi: 10.1089/fpd.2014.1758

Lee, W., and Lee, D. G. (2017). Resveratrol induces membrane and DNA disruption via pro-oxidant activity against Salmonella typhimurium. Biochem. Biophys. Res. Commun. 489, 228-234. doi: 10.1016/j.bbrc.2017.05.138

Lei, H., Ma, G., Zhong, Z., Feng, Y., Lv, X., Li, Y., et al. (2017). Whitening Anti-aging Natural Plant Composition and its Application. CN 107137301 A 20170908. Faming Zhuanli Shenqing.

Li, W., Wang, X., Yang, Y., Wu, M., Fan, P., and Qi, X. (2015). Resveratrol Containing Botanical Synergistic Complex Fungicide, its Preparation Method and Application. CN 104604861 A 20150513. Faming Zhuanli Shenqing.

Liu, Y., Zhou, J., Qu, Y., Yang, X., Shi, G., Wang, X., et al. (2016). Resveratrol antagonizes antimicrobial lethality and stimulates recovery of bacterial mutants. PLoS ONE 11:e0153023. doi: 10.1371/journal.pone.0153023

Lv, F., Liang, H., Yuan, Q., and Li, C. (2011). In vitro antimicrobial effects and mechanism of action of selected plant essential oil combinations against four food-related microorganisms. Food Res. Int. 44, 3057-3064. doi: 10.1016/j.foodres.2011.07.030

Madrigal-Perez, L. A., and Ramos-Gomez, M. (2016). Resveratrol inhibition of cellular respiration: new paradigm for an old mechanism. Int. J. Mol. Sci. 17:368. doi: $10.3390 /$ ijms 17030368

Makwana, S., Choudhary, R., Haddock, J., and Kohli, P. (2015). Invitro antibacterial activity of plant based phenolic compounds for food safety and preservation. LWT Food Sci. Technol. 62, 935-939. doi: 10.1016/j.lwt.2015.02.013

Mejlholm, O., and Dalgaard, P. (2002). Antimicrobial effect of essential oils on the seafood spoilage micro-organism Photobacterium phosphoreum in liquid media and fish products. Lett. Appl. Microbiol. 34, 27-31. doi: 10.1046/j.1472-765x.2002.01033.x

Morán, A., Gutiérrez, S., Martínez-Blanco, H., Ferrero, M. A., MonteagudoMera, A., and Rodríguez-Aparicio, L. B. (2014). Non-toxic plant metabolites regulate Staphylococcus viability and biofilm formation: a natural therapeutic strategy useful in the treatment and prevention of skin infections. Biofouling 30, 1175-1182. doi: 10.1080/08927014.2014.976207

Morinaga, N., Yahiro, K., and Noda, M. (2010). Resveratrol, a natural polyphenolic compound, inhibits cholera toxin-induced cyclic AMP accumulation in Vero cells. Toxicon 56, 29-35. doi: 10.1016/j.toxicon.2010.03.009

Oliveira, A. R., Domingues, F. C., and Ferreira, S. (2017). The influence of resveratrol adaptation on resistance to antibiotics, benzalkonium chloride, heat and acid stresses of Staphylococcus aureus and Listeria monocytogenes. Food Control 73, 1420-1425. doi: 10.1016/j.foodcont.2016.11.011

Orhan, D. D., Orhan, N., Ozcelik, B., and Ergun, F. (2009). Biological activities of Vitis vinifera L. leaves. Turk. J. Biol. 33, 341-348. doi: 10.3906/biy-0806-17

O’Toole, G. A. (2011). Microtiter dish biofilm formation assay. J. Vis. Exp. e2437. doi: $10.3791 / 2437$
Pall, M. L. (2014). Nutritional Supplements Containing Resveratrol, Nicotinic Acid, and Ribose. US 20140031299 A1 20140130. US Patent and Trademark Office.

Pangeni, R., Sahni, J. K., Ali, J., Sharma, S., and Baboota, S. (2014). Resveratrol: review on therapeutic potential and recent advances in drug delivery. Expert Opin. Drug Deliv. 11, 1285-1298. doi: 10.1517/17425247.2014.919253

Pantanella, F., Valenti, P., Natalizi, T., Passeri, D., and Berlutti, F. (2013). Analytical techniques to study microbial biofilm on abiotic surfaces: pros and cons of the main techniques currently in use. Ann. Ig. 25, 31-42. doi: 10.7416/ai.2013.1904

Paolillo, R., Carratelli, C. R., and Rizzo, A. (2011). Effect of resveratrol and quercetin in experimental infection by Salmonella enterica serovar Typhimurium. Int. Immunopharmacol. 11, 149-156. doi: 10.1016/j.intimp.2010.10.019

Pari, L., and Suresh, A. (2008). Effect of grape (Vitis vinifera L.) leaf extract on alcohol induced oxidative stress in rats. Food Chem. Toxicol. 46, 1627-1634. doi: 10.1016/j.fct.2008.01.003

Paul, B., Chereyathmanjiyil, A., Masih, I., Chapuis, L., and Benoita, A. (1998). Biological control of Botrytis cinerea causing grey mould disease of grapevine and elicitation of stilbene phytoalexin (resveratrol) by a soil bacterium. FEMS Microbiol. Lett. 165, 65-70. doi: 10.1111/j.1574-6968.1998.tb13128.x

Paulo, L., Ferreira, S., Gallardo, E., Queiroz, J. A., and Domingues, F. (2010). Antimicrobial activity and effects of resveratrol on human pathogenic bacteria. World J. Microbiol. Biotechnol. 26, 1533-1538. doi: 10.1007/s11274-010-0325-7

Plumed-Ferrer, C., Väkeväinen, K., Komulainen, H., Rautiainen, M., Smeds, A., Raitanen, J. E., et al. (2013). The antimicrobial effects of wood-associated polyphenols on food pathogens and spoilage organisms. Int. J. Food Microbiol. 164, 99-107. doi: 10.1016/j.ijfoodmicro.2013.04.001

Promgool, T., Pancharoen, O., and Deachathai, S. (2014). Antibacterial and antioxidative compounds from Cassia alata Linn. Songklanakarin. J. Sci. Technol. 36, 459-463. Available online at: http://rdo.psu.ac.th/sjstweb/journal/ 36-4/36-4-11.pdf

Qin, N., Tan, X., Jiao, Y., Liu, L., Zhao, W., Yang, S., et al. (2014). RNA-Seq-based transcriptome analysis of methicillin-resistant Staphylococcus aureus biofilm inhibition by ursolic acid and resveratrol. Sci. Rep. 4:5467. doi: 10.1038/srep05467

Reeser, R. J., Medler, R. T., Billington, S. J., Jost, B. H., and Joens, L. A. (2007). Characterization of Campylobacter jejuni biofilms under defined growth conditions. Appl. Environ. Microbiol. 73, 1908-1913. doi: 10.1128/AEM.00740-06

Renaud, S., and de Lorgeril, M. (1992). Wine, alcohol, platelets, and the French paradox for coronary heart disease. Lancet 339, 1523-1526. doi: 10.1016/0140-6736(92)91277-F

Rocha-González, H. I., Ambriz-Tututi, M., and Granados-Soto, V. (2008). Resveratrol: a natural compound with pharmacological potential in neurodegenerative diseases. CNS Neurosci. Ther. 14, 234-247. doi: $10.1111 / j .1755-5949.2008 .00045 . x$

Sardi, W. F., and Solomonson, S. H. (2005). Dietary Supplements Comprising Resveratrol and Quercetin. US 20050158376 A1 20050721. US Patent and Trademark Office.

Ser, H.-L., Tan, L. T.-H., Law, J. W.-F., Chan, K.-G., Duangjai, A., Saokaew, S., et al. (2017). Focused review: cytotoxic and antioxidant potentials of mangrovederived Streptomyces. Front. Microbiol. 8:2065. doi: 10.3389/fmicb.2017.02065

Ser, H. L., Tan, L. T. H., Palanisamy, U. D., Abd Malek, S. N., Yin, W. F., Chan, K. G., et al. (2016). Streptomyces antioxidans sp. nov., a novel mangrove soil actinobacterium with antioxidative and neuroprotective potentials. Front. Microbiol. 7:899. doi: 10.3389/fmicb.2016.00899

Sergides, C., Chirilă, M., Silvestro, L., Pitta, D., and Pittas, A. (2016). Bioavailability and safety study of resveratrol $500 \mathrm{mg}$ tablets in healthy male and female volunteers. Exp. Ther. Med. 11, 164-170. doi: 10.3892/etm.2015.2895

Seukep, J. A., Sandjo, L. P., Ngadjui, B. T., and Kuete, V. (2016). Antibacterial and antibiotic-resistance modifying activity of the extracts and compounds from Nauclea pobeguinii against Gram-negative multi-drug resistant phenotypes. BMC Complement. Altern. Med. 16:193. doi: 10.1186/s12906-016-1173-2

Shelef, L. A. (1984). Antimicrobial effects of spices. J. Food Saf. 6, 29-44. doi: 10.1111/j.1745-4565.1984.tb00477.x

Silva, Â., Duarte, A., Sousa, S., Ramos, A., and Domingues, F. C. (2016). Characterization and antimicrobial activity of cellulose derivatives films incorporated with a resveratrol inclusion complex. LWT Food Sci. Technol. 73, 481-489. doi: 10.1016/j.lwt.2016.06.043 
Subramanian, M., Goswami, M., Chakraborty, S., and Jawali, N. (2014). Resveratrol induced inhibition of Escherichia coli proceeds via membrane oxidation and independent of diffusible reactive oxygen species generation. Redox Biol. 2, 865-872. doi: 10.1016/j.redox.2014.06.007

Subramanian, M., Soundar, S., and Mangoli, S. (2016). DNA damage is a late event in resveratrol-mediated inhibition of Escherichia coli. Free Radic. Res. 50, 708-719. doi: 10.3109/10715762.2016.1169404

Surendran Nair, M., Lau, P., Belskie, K., Fancher, S., Chen, C. H., Karumathil, D. P., et al. (2016). Potentiating the heat inactivation of Escherichia coli O157:H7 in ground beef patties by natural antimicrobials. Front. Microbiol. 7:15. doi: 10.3389/fmicb.2016.00015

Taguri, T., Tanaka, T., and Kouno, I. (2006). Antibacterial spectrum of plant polyphenols and extracts depending upon hydroxyphenyl structure. Biol. Pharm. Bull. 29, 2226-2235. doi: 10.1248/bpb.29.2226

Tan, L. T.-H., Chan, K.-G., and Lee, L.-H. (2014). Application of bacteriophage in biocontrol of major foodborne bacterial pathogens. J. Mol. Biol. Mol. Imaging $1: 9$.

Tan, L. T.-H., Chan, K.-G., Khan, T. M., Bukhari, S. I., Saokaew, S., Duangjai, A., et al. (2017). Streptomyces sp. MUM212 as a source of antioxidants with radical scavenging and metal chelating properties. Front. Pharmacol. 8:276. doi: 10.3389/fphar.2017.00276

Tan, L. T., Lee, L. H., Yin, W. F., Chan, C. K., Abdul Kadir, H., Chan, K. G., et al. (2015). Traditional uses, phytochemistry, and bioactivities of Cananga odorata (Ylang-Ylang). Evid. Based Complement. Alternat. Med. 2015:896314. doi: 10.1155/2015/896314

Tang, C., Hoo, P. C., Tan, L. T., Pusparajah, P., Khan, T. M., Lee, L. H., et al. (2016). Golden needle mushroom: a culinary medicine with evidenced-based biological activities and health promoting properties. Front. Pharmacol. 7:474. doi: 10.3389/fphar.2016.00474

Tassou, C. C., Drosinos, E. H., and Nychas, G. J. (1995). Effects of essential oil from mint (Mentha piperita) on Salmonella enteritidis and Listeria monocytogenes in model food systems at 4 degrees and 10 degrees C. J. Appl. Bacteriol. 78, 593-600. doi: 10.1111/j.1365-2672.1995.tb03104.x

Tegos, G., Stermitz, F. R., Lomovskaya, O., and Lewis, K. (2002). Multidrug pump inhibitors uncover remarkable activity of plant antimicrobials. Antimicrob. Agents Chemother. 46, 3133-3141. doi: 10.1128/AAC.46.10.3133-3141.2002

Tellez, G., Rodríguez-Fragoso, L., Kuttappan, V., Kallapura, G., Velasco, X., and Menconi, A. (2013). Probiotics for human and poultry use in the control of gastrointestinal disease: a review of real-world experiences. Altern. Integr. Med. 2:118. doi: 10.4172/2327-5162.1000118

Tomé-Carneiro, J., Larrosa, M., González-Sarrías, A., Tomás-Barberán, F. A., García-Conesa, M. T., and Espín, J. C. (2013). Resveratrol and clinical trials: the crossroad from in vitro studies to human evidence. Curr. Pharm. Des. 19, 6064-6093. doi: 10.2174/13816128113199990407

Walle, T., Hsieh, F., Delegge, M. H., Oatis, J. E. Jr., and Walle, U. K. (2004). High absorption but very low bioavailability of oral resveratrol in humans. Drug Metab. Dispos. 32, 1377-1382. doi: 10.1124/dmd.104.000885

Xi, Y., Sullivan, G. A., Jackson, A. L., Zhou, G. H., and Sebranek, J. G. (2011). Use of natural antimicrobials to improve the control of Listeria monocytogenes in a cured cooked meat model system. Meat Sci. 88, 503-511. doi: 10.1016/j.meatsci.2011.01.036

Yong, Y. L., Tan, L. T.-H., Ming, L. C., Chan, K.-G., Lee, L.-H., Goh, B.-H., et al. (2017). The effectiveness and safety of topical capsaicin in postherpetic neuralgia: a systematic review and meta-analysis. Front. Pharmacol. 7:538. doi: 10.3389/fphar.2016.00538

Yoo, J. H., and Kim, D. J. (2013). Antibacterial Composition Spray Container Cleaning Foot. KR 2013032047 A 20130401. Kongkae Taeho Kongbo.

Zheng, J., and Ramirez, V. D. (2000). Inhibition of mitochondrial proton F0F1ATPase/ATP synthase by polyphenolic phytochemicals. Br. J. Pharmacol. 130, 1115-1123. doi: 10.1038/sj.bjp.0703397

Zhu, J., Pan, A., Chen, S., and Lu, Z. (2016). An Antibacterial Hand Cleanser. CN 106214563 A 20161214. Faming Zhuanli Shenqing.

Zini, R., Morin, C., Bertelli, A., Bertelli, A. A., and Tillement, J. P. (1999). Effects of resveratrol on the rat brain respiratory chain. Drugs Exp. Clin. Res. 25, 87-97.

Conflict of Interest Statement: The authors declare that the research was conducted in the absence of any commercial or financial relationships that could be construed as a potential conflict of interest.

Copyright (c) 2018 Ma, Tan, Chan, Yap, Pusparajah, Chuah, Ming, Khan, Lee and Goh. This is an open-access article distributed under the terms of the Creative Commons Attribution License (CC BY). The use, distribution or reproduction in other forums is permitted, provided the original author(s) and the copyright owner are credited and that the original publication in this journal is cited, in accordance with accepted academic practice. No use, distribution or reproduction is permitted which does not comply with these terms. 